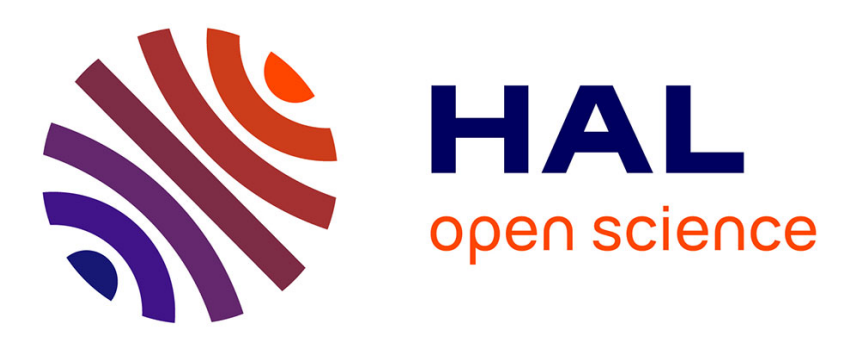

\title{
Liouville type results for local minimizers of the micromagnetic energy
}

François Alouges, Giovanni Di Fratta, Benoit Merlet

\section{To cite this version:}

François Alouges, Giovanni Di Fratta, Benoit Merlet. Liouville type results for local minimizers of the micromagnetic energy. Calculus of Variations and Partial Differential Equations, 2014, 53 (3-4), pp.525-560. 10.1007/s00526-014-0757-2 . hal-01584850

\section{HAL Id: hal-01584850 \\ https://hal.science/hal-01584850}

Submitted on 10 Sep 2017

HAL is a multi-disciplinary open access archive for the deposit and dissemination of scientific research documents, whether they are published or not. The documents may come from teaching and research institutions in France or abroad, or from public or private research centers.
L'archive ouverte pluridisciplinaire HAL, est destinée au dépôt et à la diffusion de documents scientifiques de niveau recherche, publiés ou non, émanant des établissements d'enseignement et de recherche français ou étrangers, des laboratoires publics ou privés. 


\title{
Liouville type results for local minimizers of the micro- magnetic energy.
}

\author{
By François Alouges, Giovanni Di Fratta, Benoit Merlet
}

\begin{abstract}
We study local minimizers of the micromagnetic energy in small ferromagnetic $3 \mathrm{~d}$ convex particles for which we justify the Stoner-Wohlfarth approximation: given a uniformly convex shape $\Omega \subset \mathbf{R}^{3}$, there exist $\delta_{c}>0$ and $C>0$ such that for $0<\delta \leq \delta_{c}$ any local minimizer $\mathbf{m}$ of the micromagnetic energy in the particle $\delta \Omega$ satisfies $\|\nabla \mathbf{m}\|_{L^{2}} \leqslant C \delta^{2}$. In the case of ellipsoidal particles we strengthen this result by proving that, for $\delta$ small enough, local minimizers are exactly spatially uniform.

This last result extends W.F. Brown's fundamental theorem for fine 3d ferromagnetic particles [Brown (1968), Di Fratta et al. (2011)] which states the same result but only for global minimizers.

As a by-product of the method that we use, we establish a new Liouville type result for locally minimizing $p$-harmonic maps with values into a closed subset of a Hilbert space. Namely, we establish that in a smooth uniformly convex domain of $\mathbf{R}^{d}$ any local minimizer of the $p$-Dirichlet energy $(p>1$, $p \neq d)$ is constant.
\end{abstract}

Keywords: Micromagnetism, Single-domain particles, Harmonic Maps, Local Minimizers.

AMS Cl.: 35B35, 35B53, 49K20, 49K40, 49S05, 74G65, 82D40.

\section{Introduction and main results}

Micromagnetism as introduced by Landau-Lifshitz and Brown describes the magnetization states inside a ferromagnetic body below the Curie temperature (see [citerLL], [7] and the textbook [20]). According to this theory, the magnetization in a ferromagnetic sample occupying the domain $\Omega \subset \mathbf{R}^{3}$ is modeled by a vector field $\mathbf{m}: \Omega \rightarrow \mathbf{R}^{3}$, of constant magnitude $M_{s}$, the saturation magnetization, that we assume equal to 1 after normalization. The (static) theory then states that observed magnetization distributions are local minimizers of the micromagnetic energy

$$
\mathcal{E}(\mathbf{m} ; \Omega):=\frac{l_{\mathrm{ex}}^{2}}{2} \int_{\Omega}|\nabla \mathbf{m}(x)|^{2} d x+\int_{\Omega} \psi(\mathbf{m}(x)) d x-\frac{1}{2} \int_{\Omega} \mathbf{h}_{d}[\mathbf{m} ; \Omega](x) \cdot \mathbf{m}(x) d x .
$$

a) The first term is called exchange energy and $l_{\mathrm{ex}}$ is the exchange length. This term penalizes brutal variations of the magnetization.

b) The second term combines anisotropy effects and the action of an external field $\mathbf{h}_{\text {ext }}$

$$
\psi(\mathbf{u})=A_{\text {anis }}(\mathbf{u})-\mathbf{h}_{\mathrm{ext}} \cdot \mathbf{u},
$$

where $A_{\text {anis }}: \mathbb{S}^{2} \rightarrow \mathbf{R}^{+}$is a non negative function that vanishes at the so-called easy directions. When merged with the energy due to the external field, the corresponding contribution favors directions of magnetization which minimize $\psi$.

c) The last term, called stray field energy is a non local self-interaction energy. The vector field $\mathbf{h}_{d}[\mathbf{m}$; $\Omega$ ] (usually called stray field or demagnetizing field) represents the magnetic field generated by magnetization distribution $\mathbf{m}$ itself through Maxwell equations. From a mathematical point of view the simplest and shortest way to define $\mathbf{h}_{d}[\mathbf{m} ; \Omega]$ is to extend $\mathbf{m}$ by 0 in $\mathbf{R}^{3} \backslash \bar{\Omega}$ by setting $\mathbf{m}_{0}:=\mathbf{1}_{\Omega} \mathbf{m}$. The stray field is then defined as the opposite of the projection of $\mathbf{m}_{0}$ in $L^{2}\left(\mathbf{R}^{3}, \mathbf{R}^{3}\right)$ on the closed subspace,

$$
V:=\left\{\nabla \mathbf{v}: \mathbf{v} \in \mathcal{D}^{\prime}\left(\mathbf{R}^{3}, \mathbf{R}\right), \nabla \mathbf{v} \in L^{2}\left(\mathbf{R}^{3}, \mathbf{R}^{3}\right)\right\}
$$


In particular, we see that the operator $\mathbf{m} \mapsto \mathbf{h}_{d}[\mathbf{m} ; \Omega]$ is a non-local pseudo-differential operator of order 0 . By properties of the orthogonal projection, the stray field energy rewrites

$$
-\frac{1}{2} \int_{\Omega} \mathbf{h}_{d}[\mathbf{m} ; \Omega] \cdot \mathbf{m}=-\frac{1}{2} \int_{\mathbf{R}^{3}} \mathbf{h}_{d}[\mathbf{m} ; \Omega] \cdot \mathbf{m}_{0}=\frac{1}{2} \int_{\mathbf{R}^{3}}\left|\mathbf{h}_{d}[\mathbf{m} ; \Omega]\right|^{2},
$$

and we have the bounds

$$
0 \leq-\frac{1}{2} \int_{\Omega} \mathbf{h}_{d}[\mathbf{m} ; \Omega] \cdot \mathbf{m} \leq \frac{1}{2}\|\mathbf{m}\|_{L^{2}}^{2}=\frac{|\Omega|}{2} .
$$

This energy is non-negative and vanishes if and only if $\mathbf{m}_{0}$ belongs to $V^{\perp}$, that is, by De Rham's Theorem, if and only if $\mathbf{m}_{0}$ is divergence free in the sense of distributions (this amounts formally to $\nabla \cdot \mathbf{m} \equiv 0$ in $\Omega$ and $\mathbf{m} \cdot \mathbf{n} \equiv 0$ on $\partial \Omega$ ). The components of $\nabla \cdot \mathbf{m}_{0}$ induced by $\mathbf{1}_{\Omega} \nabla \cdot \mathbf{m}$ and $\mathbf{1}_{\partial \Omega} \mathbf{m} \cdot \mathbf{n}$ are respectively called volume and surface charges.

Existence of a minimizer of the micromagnetic energy is easily obtained by the direct method of the calculus of variation (at least when $\psi$ is lower semi-continuous and $\Omega \subset \mathbf{R}^{3}$ is a non empty open set with finite volume).

Here, we are interested in the behavior of the magnetization when the shape of the ferromagnetic sample is fixed and its size is comparable to the exchange length. For this, we introduce a reference domain $\Omega$ with unit diameter that we rescale by setting $\Omega_{\delta}:=\delta \Omega$ where $\delta>0$ is a (small) parameter. Similarly, for any magnetization distribution $\mathbf{m}_{\delta}$ defined in the physical domain $\Omega_{\delta}$ we set $\mathbf{m}(x)=\mathbf{m}_{\delta}(\delta x)$ for $x \in \Omega$, the reference domain. With this change of variable, we have $\delta \nabla \mathbf{m}_{\delta}(\delta x)=\nabla \mathbf{m}(x)$ while $\mathbf{h}_{d}\left[\mathbf{m}_{\delta} ; \Omega_{\delta}\right](\delta x)=\mathbf{h}_{d}[\mathbf{m}$; $\Omega](x)$. Therefore the three energy terms scale as

and

$$
\begin{aligned}
& \int_{\Omega_{\delta}}\left|\nabla \mathbf{m}_{\delta}\right|^{2}\left(x_{\delta}\right) d x_{\delta}=\delta \int_{\Omega}|\nabla \mathbf{m}|^{2}(x) d x \\
& \int_{\Omega_{\delta}} \psi\left(\mathbf{m}_{\delta}\left(x_{\delta}\right)\right) d x_{\delta}=\delta^{3} \int_{\Omega} \psi(\mathbf{m}(x)) d x
\end{aligned}
$$

$$
\int_{\Omega_{\delta}} \mathbf{h}_{d}\left[\mathbf{m}_{\delta} ; \Omega_{\delta}\right]\left(x_{\delta}\right) \cdot \mathbf{m}_{\delta}\left(x_{\delta}\right) d x_{\delta}=\delta^{3} \int_{\Omega} \mathbf{h}_{d}[\mathbf{m} ; \Omega] \cdot \mathbf{m} d x .
$$

Introducing the non-dimensional parameter $\varepsilon:=\delta / l_{\mathrm{ex}}$, we get

$$
\frac{1}{\varepsilon l_{\mathrm{ex}}^{3}} \mathcal{E}\left(\mathbf{m}_{\delta} ; \Omega_{\delta}\right)=\mathcal{D}_{\varepsilon}(\mathbf{m}):=\frac{1}{2} \int_{\Omega}|\nabla \mathbf{m}(x)|^{2} d x+\varepsilon^{2}\left(\frac{1}{2} \int_{\mathbf{R}^{3}}\left|\mathbf{h}_{d}[\mathbf{m} ; \Omega]\right|^{2}+\int_{\Omega} \psi(\mathbf{m})\right)
$$

that we rewrite under the form

$$
\mathcal{D}_{\varepsilon}(\mathbf{m}):=\mathcal{D}(\mathbf{m})+\varepsilon^{2} \mathcal{F}(\mathbf{m}), \quad \text { with } \quad \mathcal{F}(\mathbf{m}):=\frac{1}{2} \int_{\mathbf{R}^{3}}\left|\mathbf{h}_{d}[\mathbf{m} ; \Omega]\right|^{2}+\int_{\Omega} \psi(\mathbf{m}) .
$$

For $\varepsilon \gg 1$, i.e. for samples much larger than the exchange length, the prominent terms in the energy are the stray field energy and the anisotropy. Minimizing magnetization distributions are not uniform in these situations because constant magnetizations induce large surface charges. The typical observed behavior is in fact a partition of the sample into regions called domains where the magnetization is almost constant separated by thin layers called domain walls of thickness comparable to $l_{\text {ex }}$ where the energy concentrates.

For fine particles $\varepsilon \ll 1$, it is expected that the cost of domain walls exceeds the cost of surface charges. In this case, the magnetization is almost uniform inside the body and the particle is said to be single-domain. At the limit, according to the Stoner-Wohlfarth theory [30] the magnetization is considered as spatially uniform in the particle, that is,

$$
\mathbf{m} \in U\left(\Omega, \mathbb{S}^{2}\right):=\left\{\mathbf{u}: \Omega \rightarrow \mathbb{S}^{2}: \exists \sigma \in \mathbb{S}^{2} \text { such that } \mathbf{u} \equiv \sigma \text { almost everywhere in } \Omega\right\} .
$$

In this case, the micromagnetic energy of $\mathbf{u} \equiv \sigma \in U\left(\Omega, \mathbb{S}^{2}\right)$ reduces to

$$
\mathcal{D}_{\varepsilon}(\mathbf{u})=\varepsilon^{2}|\Omega|\left(\frac{1}{2} \sigma^{T} \cdot \mathbf{N}_{\mathrm{eff}} \sigma+\psi(\sigma)\right)
$$


where the effective demagnetizing tensor $\mathbf{N}_{\text {eff }}$ is the $3 \times 3$ matrix defined by,

$$
\mathbf{N}_{\mathrm{eff}} \sigma=-\frac{1}{|\Omega|} \int_{\Omega} \mathbf{h}_{\mathrm{d}}[\mathbf{u}](x) d x .
$$

The tensor $\mathbf{N}_{\text {eff }}$ inherits the properties of $-\mathbf{h}_{d}[\cdot, \Omega]$ as a continuous orthogonal projector of $L^{2}\left(\Omega, \mathbf{R}^{3}\right)$. In particular $\mathbf{N}_{\text {eff }}$ is a non negative symmetric matrix and its eigenvalues are bounded by 1 . Also notice that since $\mathbf{m} \mapsto-\mathbf{h}_{d}[\mathbf{m}, \Omega]$ is a linear pseudo-differential operator of order 0 , the coefficients of $\mathbf{N}_{\text {eff }}$ only depend on the shape of $\Omega$ and not on its diameter.

Let us state a simple result supporting the Stoner-Wohlfarth approximation: in small particles, minimizers of the micromagnetic energy are almost constant.

Proposition 1. Let $\Omega$ be an open subset of $\mathbf{R}^{3}$ with a finite volume $|\Omega|$, let $\psi: \mathbb{S}^{2} \rightarrow \mathbf{R}$ be lower semicontinuous and let $\varepsilon>0$. Then if $\mathbf{m}$ is a global minimizer of $\mathcal{D}_{\varepsilon}$ in $H^{1}\left(\Omega, \mathbb{S}^{2}\right)$, it satisfies

$$
\|\nabla \mathbf{m}\|_{L^{2}}^{2} \leq|\Omega| \varepsilon^{2} .
$$

Proof. If $\mathbf{m}$ is a minimizer, then $\mathcal{D}_{\varepsilon}(\mathbf{m}) \leq \varepsilon^{2} \mathcal{F}(\mathbf{u})$ for any $\mathbf{u} \equiv \sigma \in U\left(\Omega, \mathbb{S}^{2}\right)$. Choosing $\sigma \in \mathbb{S}^{2}$ minimizing $\psi$, we have $\|\nabla \mathbf{m}\|_{L^{2}}^{2} \leq 2 \varepsilon^{2}(\mathcal{F}(\mathbf{u})-\mathcal{F}(\mathbf{m})) \leq \varepsilon^{2}|\Omega| \sigma^{T} \cdot \mathbf{N}_{\text {eff }} \sigma \leq|\Omega| \varepsilon^{2}$.

Moreover, A. De Simone established in [10] that for $\varepsilon>0$ small, the magnetization can not substantially decrease its energy by moving away from the set of constant maps.

Proposition 2. (Corollary of Proposition 3.4. in [10])

$$
\lim _{\varepsilon \downarrow 0} \min _{\mathbf{m} \in H^{1}\left(\Omega, \mathbb{S}^{2}\right)} \frac{1}{\varepsilon^{2}} \mathcal{D}_{\varepsilon}(\mathbf{m})=\min _{\mathbf{u} \in U\left(\Omega, \mathbb{S}^{2}\right)} \mathcal{F}(\mathbf{u}) .
$$

The Stoner-Wohlfarth approximation is almost never exact. Indeed, assume that $\mathbf{u} \equiv \sigma \in U\left(\Omega, \mathbb{S}^{2}\right)$ is a minimizer or even a critical point of $\mathcal{D}_{\varepsilon}$ in $H^{1}\left(\Omega, \mathbb{S}^{2}\right)$, the associated Euler-Lagrange equation at this point reads,

$$
-\mathbf{h}_{d}[\mathbf{u} ; \Omega](x)+D \psi(\sigma) \in \operatorname{span}\{\sigma\} \text { in } \Omega .
$$

Consequently, in the plane $\sigma^{\perp}$, the components of $\mathbf{h}_{d}[\mathbf{u}, \Omega]$ should be uniform in $\Omega$. This turns out to be wrong for general domains with the notable exception of $\Omega$ being a solid sphere or even a solid ellipsoid. Indeed, in these latter cases, a well known result of potential theory ([22], [25]), states that the stray field induced by uniform magnetizations is also uniform inside $\Omega$. For these special geometries the effective stray field is point-wise related to $\mathbf{u}$.

Proposition 3. (Maxwell [25]) If $\Omega$ is a solid ellipsoid then, the linear mapping $\mathbf{u} \mapsto-\mathbf{h}_{d}[\mathbf{u} ; \Omega]_{\mid \Omega}$ maps $U\left(\Omega, S^{2}\right)$ into itself (i.e. $-\mathbf{h}_{d}[\mathbf{u}]=\mathbf{N}_{\mathrm{eff}} \sigma$ in $\Omega$ for $\mathbf{u} \equiv \sigma$ ).

Remark 4. Explicit formulas are known for the coefficients of $\mathbf{N}_{\text {eff }}$ in general ellipsoids (see [25], [26], or [20] chapter 3.2). In particular, in this case, we have $\operatorname{Tr} \mathbf{N}_{\text {eff }}=1$.

If $\Omega$ is the solid rotation ellipsoid $\left\{x \in \mathbf{R}^{3}:\left(x_{1} / a\right)^{2}+\left(x_{2} / b\right)^{2}+\left(x_{3} / b\right)^{2}<R^{2}\right\}$, then the eigenvalues of $\mathbf{N}_{\text {eff }}$ in the standard basis are $\lambda_{1}, \lambda_{2}=\lambda_{3} \in(0,1)$ with $\lambda_{1}+2 \lambda_{2}=1$. The extremal coefficients are obtained in the limit of oblate ellipsoids: $\left(\lambda_{1}, \lambda_{2}, \lambda_{3}\right) \rightarrow(1,0,0)$ as $b / a \rightarrow \infty$. For prolate ellipsoids, we have $\left(\lambda_{1}, \lambda_{2}, \lambda_{3}\right) \rightarrow(0,1 / 2,1 / 2)$ as $b / a \rightarrow 0$.

The Fundamental Theorem for fine ferromagnetic particles of W.F. Brown is stated in this setting:

Theorem 5. (Brown [6]: solid sphere case - Aharoni [1]: prolate spheroids - [11](see also [2]): general ellipsoids)

Assume that $\Omega$ is a solid ellipsoid of unit diameter and that $\psi$ is of class $C^{1}$ on $\mathbb{S}^{2}$. There exists $\varepsilon_{c}>0$ such that for every $\varepsilon \in\left[0, \varepsilon_{c}\right)$, any minimizer of $\mathcal{D}_{\varepsilon}$ in $H^{1}\left(\Omega, \mathbb{S}^{2}\right)$ is uniform in $\Omega$. 
In fact, in the above references, the result is established assuming that $A_{\text {anis. }}$ is a second order polynomial, $\left(\psi(\sigma)=\psi_{0}+\mathbf{H} \cdot \sigma+(1 / 2) \sigma^{T} \cdot A \sigma\right)$. For this reason, we provide the reader with a general proof of Theorem 5 in Section 2.2.

Remark 6. The proof of Theorem 5 gives an explicit lower bounds for $\varepsilon_{c}$. We can also derive an upper bound by a linear stability analysis of the uniform magnetizations. Unfortunately, these bounds are not sharp (and do not match). The critical value $\varepsilon_{c}$ is not known explicitly, but can be determined by numerical means (see e.g. [3], [4]). This remark also applies to the constants introduced in our main result below.

Remark 7. When $\psi$ is a second order polynomial function, the minimizers of $\mathcal{D}_{\varepsilon}$ in $U\left(\Omega, \mathbb{S}^{2}\right)$ are easily deduced from the coefficients of $\psi$ and $\mathbf{N}_{\text {eff }}$. For example, if $\psi \equiv 0$ and if $\Omega$ is the solid ellipsoid defined by $\left\{x \in \mathbf{R}^{3}:\left(x_{1} / a\right)^{2}+\left(x_{2} / b\right)^{2}+\left(x_{3} / c\right)^{2}<R^{2}\right\}$ with semi-axes $a \geq b \geq c>0$, these minimizers are

i. all the elements of $U\left(\Omega, S^{2}\right)$ in the case $a=b=c$ (sphere);

ii. the elements of the circle $U\left(\Omega, S^{2} \cap \operatorname{span}\left\{\mathbf{e}_{1}, \mathbf{e}_{2}\right\}\right)$ if $a=b>c$ (prolate ellipsoid);

iii. the two vectors $\pm \mathbf{e}_{1}$ if $a>b \geq c$ (elongated ellipsoid).

Proposition 1 and Brown's Theorem do not describe all the stable observable configurations in ellipsoidal domains, since these results do not rule out the existence of non uniform local minimizers of $\mathcal{D}_{\varepsilon}$. The main contribution of this paper consists in filling this gap, at least for smooth uniformly convex particles.

Theorem 8. Let $\psi \in C^{2}\left(\mathbb{S}^{2}, \mathbf{R}\right)$ and let $\Omega \subset \mathbf{R}^{3}$ be a $C^{2}$ uniformly convex domain with unit diameter. Let $\varepsilon>0$ and assume that $\mathbf{m}$ is a local minimizer of $\mathcal{D}_{\varepsilon}$ in $H^{1}\left(\Omega, \mathbb{S}^{2}\right)$, i.e., there exists $\eta>0$ such that for every $\mathbf{p} \in H^{1}\left(\Omega, \mathbb{S}^{2}\right)$,

$$
\|\mathbf{p}-\mathbf{m}\|_{H^{1}} \leqslant \eta \Longrightarrow \mathcal{D}_{\varepsilon}(\mathbf{p}) \geq \mathcal{D}_{\varepsilon}(\mathbf{m})
$$

Then

i. there exist $\varepsilon_{\mathcal{F}}>0$ and $C_{\mathcal{F}} \geq 0$ only depending on $\Omega$ and $\psi$ such that:

$$
\varepsilon<\varepsilon_{\mathcal{F}} \Longrightarrow\|\nabla \mathbf{m}\|_{L^{2}(\Omega)} \leq C_{\mathcal{F}} \varepsilon^{2} .
$$

ii. if moreover $\Omega$ is an ellipsoid, there exists $\varepsilon_{\mathcal{F}}^{\prime}>0$ which only depends on $\Omega$ and $\psi$ such that

$$
\varepsilon<\varepsilon_{\mathcal{F}}^{\prime} \Longrightarrow \mathbf{m} \in U\left(\Omega, \mathbb{S}^{2}\right)
$$

The uniformity in space of locally minimizing magnetizations in small ellipsoidal particles (stated in the second part) was conjectured by Brown himself [6].

The first part of the Theorem implies $\mathcal{D}(\mathbf{m})=O\left(\varepsilon^{4}\right)$ for local minimizers of $\mathcal{D}_{\varepsilon}$ in a smooth uniformly convex particle. Thus the main contribution of the energy comes from the lower order term $\varepsilon^{2} \mathcal{F}(\mathbf{m})$ which leads to $\mathcal{D}_{\varepsilon}(\mathbf{m})=O\left(\varepsilon^{2}\right)$. This rules out the existence of high energy local minimizers, in particular, any family $\left\{\mathbf{m}_{\varepsilon}\right\}_{\varepsilon<\varepsilon_{\mathcal{F}}}$ of local minimizers of $\left\{\mathcal{D}_{\varepsilon}\right\}_{\varepsilon<\varepsilon_{\mathcal{F}}}$ converges up to extraction towards a critical point of $\mathcal{F}$ in $U\left(\Omega, \mathbb{S}^{2}\right)$.

In the small particle limit $\varepsilon=0$, we may believe that, for finding the observed magnetization distributions, it is sufficient to replace the Dirichlet energy by the constraint $\mathbf{m} \in U\left(\Omega, \mathbb{S}^{2}\right)$ and look for local minimizers of $\mathcal{F}$ in this set. It is indeed true that when $\mathcal{F}$ admits an isolated local minimizer $\mathbf{u}$ in the set of uniform magnetizations then there exists a family of magnetizations $\left\{\mathbf{m}_{\varepsilon}\right\}_{\varepsilon<\varepsilon_{0}}$ such that $\mathbf{m}_{\varepsilon}$ is a local minimizer of $\mathcal{D}_{\varepsilon}$ and $\mathbf{m}_{\varepsilon} \rightarrow \mathbf{u}$ in $L^{2}$ as $\varepsilon \downarrow 0$ (see [10] Theorem 4.3).

On the other hand, the situation is more complex when $\mathcal{F}$ admits a continuum of local minimizers in $U\left(\Omega, \mathbb{S}^{2}\right)$, since in this case $\mathcal{D}_{\varepsilon}$ may admit only finitely many local minimizers. This phenomenon called configurational anisotropy is due to the slight deviation of $\mathbf{m}_{\varepsilon}$ from the set of uniform magnetizations (see [9] and the rigorous analysis in [29] for prism-shaped particles with $\mathbb{D}_{4}$ symmetry). 


\subsection{Locally minimizing $p$-harmonic maps.}

In the proof of our main result, we establish a Liouville type result for harmonic maps that we believe of independent interest. Since it does not make the proof more cumbersome, we state our result in the setting of $p$-harmonic maps with values into a general Hilbert space.

Let $\Omega \subset \mathbf{R}^{d}$ be a bounded open set, $p>1$ and $H$ be a Hilbert space. The $p$-Dirichlet energy of a mapping $\mathbf{m} \in W^{1, p}(\Omega, H)$ is defined as

$$
\mathcal{E}(\mathbf{m})=\mathcal{E}(\mathbf{m} ; \Omega):=\frac{1}{p} \int_{\Omega}|\nabla \mathbf{m}(\mathbf{x})|_{H}^{p} \mathrm{~d} \mathbf{x}
$$

where $|\cdot|_{H}$ stands for the norm in $H$. Given a closed subset $\mathcal{S}$ of $H$, we define $W^{1, p}(\Omega, \mathcal{S})$ to be the set

$$
W^{1, p}(\Omega, \mathcal{S})=\left\{\mathbf{m} \in W^{1, p}(\Omega, H) \text { such that } \mathbf{m}(x) \in \mathcal{S} \text { for almost every } x \in \Omega\right\} .
$$

We first address the question of whether local minimizers of $\mathcal{E}$ in $W^{1, p}(\Omega, \mathcal{S})$ are constant vector fields (i.e. $\mathbf{m} \equiv \sigma \in \mathcal{S}$ a.e. in $\Omega$ ); in this case local minimizers would be global minimizers.

If $\mathcal{S}$ were star-shaped with respect to some point $\sigma$, it is pretty obvious that a local minimizer $\mathbf{m}$ of $\mathcal{E}$ in $W^{1, p}(\Omega, \mathcal{S})$ should be a constant vector field (just compare $\mathcal{E}(\mathbf{m})$ to $\mathcal{E}((1-\varepsilon) \mathbf{m}+\varepsilon \sigma)$ for $\left.\varepsilon \downarrow 0\right)$. In the general case, such variations using convex combinations are not possible, and this is a classical difficulty for the study of the regularity of harmonic maps.

When $\mathcal{S}$ is a smooth manifold, critical points of $\mathcal{E}$ in $W^{1, p}(\Omega, \mathcal{S})$ are called p-harmonic maps or simply harmonic maps in the case $p=2$. Such a map satisfies, at least formally, the Euler Lagrange equations:

$$
-\nabla \cdot\left(|\nabla \mathbf{m}|_{H}^{p-2} \nabla \mathbf{m}\right) \in T_{\mathbf{m}(x)} \mathcal{S} \quad \text { a.e. } \operatorname{in} \Omega .
$$

We prove the following result.

Theorem 9. Let $\Omega \subset \mathbf{R}^{d}$ be a bounded open set, let $p>1$ and let $\mathcal{S}$ be a closed subset of a Hilbert space $H$. Assume that $\mathbf{m}$ is local minimizer of $\mathcal{E}$ in $W^{1, p}(\Omega, \mathcal{S})$. We have,

i. if $p>d$ and $\Omega$ is star-shaped, then $\mathbf{m}$ is constant;

ii. if $p=d$ and $\Omega$ is star-shaped, then $\nabla \mathbf{m}$ is supported in $\lambda \Omega$ for some $\lambda<1$;

iii. if $p<d$ and if $\Omega$ is a $C^{2}$ uniformly convex domain then $\mathbf{m}$ is constant.

Remark 10. In case ii $(p=d)$, if we knew that $\mathbf{m}$ were analytic, we would be in a position to conclude, using the unique continuation property, that $\mathbf{m}$ is uniform on $\Omega$. Such a situation occurs when $d=p=2$ and $\mathcal{S} \subset \mathbf{R}^{N}$ is an analytic embedded compact manifold. In this case a Theorem of Morrey [24] states that $\mathbf{m}$ is Hölder continuous. This allows us to localize in the target manifold, i.e. if $U$ is a neighborhood of $\mathbf{m}(x)$ in $\mathcal{S}$, then there exists a neighborhood $\omega \subset \Omega$ of $x$ such that $\mathbf{m}(\omega) \subset U$. Using analytic local charts $\psi: U \subset \mathcal{S} \rightarrow \mathbf{R}^{2}$, we see that $\psi \circ \mathbf{m}$ is a critical point of a coercive functional of the form $\int_{\omega} \nabla \mathbf{u}(x)^{T} \cdot A(\mathbf{u}(x)) \nabla \mathbf{u}(x) d x$ defined for $\mathbf{u} \in H^{1}\left(\omega, \mathbf{R}^{2}\right)$. The associated Euler Lagrange equations now read as a non degenerate, quasilinear elliptic system with analytic coefficients. The general regularity theory for these systems yields the analyticity of $\psi \circ \mathbf{m}$. Hence $\mathbf{m}$ is analytic and thanks to $i$, spatially uniform.

Remark 11. In fact, we establish iii under a slightly weaker convexity assumption on $\Omega$. The stated assumption amounts to ask for the second fundamental form $A_{y}$ on $\partial \Omega$ to be uniformly coercive, i.e. there exists $c>0$ such that $A_{y}(\mathbf{v}, \mathbf{v}) \geq c|\mathbf{v}|^{2}$ for every $y \in \partial \Omega$ and every $\mathbf{v} \in T_{y} \partial \Omega$. We can relax this hypothesis by assuming that $\Omega \subset \mathbf{R}^{d}$ is bounded, convex and of class $C^{2}$ and that $A(y)$ is coercive for almost every $y$ in $\partial \Omega$.

There is a huge literature on the qualitative theory of harmonic maps dealing with existence, regularity and singularity issues. We refer the reader to the review papers [12], [13], [14], [15], [19], [31] and more recently [21]. The regularity of $p$-harmonic maps has also been investigated, see e.g. [16], [17], [18] and [23]. The proof of Theorem 9 relies on ideas from the interior regularity theory of minimizing harmonic maps, in particular we use the notion of inner variation and a refined version of the monotonicity formula of Schoen and Uhlenbeck [28]. However the present paper is essentially self-contained, mainly because we need a specific treatment of the boundary. 
In Section 2, we state a general stability result for perturbations $\mathcal{D}_{\varepsilon}=\mathcal{D}+\varepsilon^{2} \mathcal{F}$ of the Dirichlet energy: Theorem 14, we show that the micromagnetic energy satisfies the relevant hypotheses and establish that Theorem 8 follows from Theorem 14. We also prove Theorem 5 at the end of the Section. We establish Theorem 9 and Theorem 14 in Sections 3 and 4, respectively. Eventually, in Section 5 we discuss open questions and possible generalizations of our results.

\subsection{Notation}

For a mapping $\mathbf{m}: \Omega \rightarrow H$, we denote by $\langle\mathbf{m}\rangle=\frac{1}{|\Omega|} \int_{\Omega} \mathbf{m} \in H$ the mean value of $\mathbf{m}$ over $\Omega$.

In order to ease possible further studies concerning the dependency of the constants appearing in Theorem 8 with respect to $\Omega$ and $\psi$, we keep track of the constants in our estimates. In particular, we will use the Poincaré inequality for functions with vanishing mean value. Let us recall that the value of the Poincaré constant admits a universal bound in convex domains with prescribed diameter.

Proposition 12. (Poincaré inequality, see [5], [27]) Let $\Omega \subset \mathbf{R}^{d}$ be a bounded convex open set. There exists $C_{P} \geq 0$ such that,

$$
\|f-\langle f\rangle\|_{L^{2}} \leq C_{P}\|\nabla f\|_{L^{2}} \quad \text { for every } f \in H^{1}(\Omega) .
$$

Moreover, the optimal constant satisfies $C_{P} \leq \delta / \pi$ where $\delta$ is the diameter of $\Omega$.

We will also make use of the following variation of the Poincare inequality when $\Omega$ is a smooth convex domains, which contains 0 .

Proposition 13. (interior-boundary Poincaré inequality) Let $\Omega \subset \mathbf{R}^{d}$ be a bounded smooth convex domain, There exists $C_{P}^{\prime} \geq 0$ such that

$$
\left(\int_{\Omega \times \partial \Omega}|f(x)-f(y)|^{2}(\mathbf{n}(y) \cdot y) d x d \mathcal{H}^{d-1}(y)\right)^{1 / 2} \leq C_{P}^{\prime} \sqrt{\Omega}\|\nabla f\|_{L^{2}} \quad \text { for every } f \in H^{1}(\Omega),
$$

and $C_{P}^{\prime} \leq \sqrt{2}(1+(d+1) / \pi) \delta$, where $\delta$ is the diameter of $\Omega$.

For the convenience of the reader, we establish (3) in Appendix A.

\section{A general stability/rigidity result. Proof of Theorems 5 and 8}

We obtain Theorem 8 as a particular case of the more general Theorem 14 given below which concerns more general energies $\mathcal{D}_{\varepsilon}:=\mathcal{D}+\varepsilon^{2} \mathcal{F}$ defined for functions $\mathbf{m} \in H^{1}(\Omega, \mathcal{S})$ where $\mathcal{S}$ is a closed subset of the Hilbert $H$. Let us list the relevant hypotheses for this result.

First, since our method relies on the tools developed for the proof of Theorem 9. iii., we need:

(H1) $\Omega$ is a $C^{2}$, uniformly convex domain of $\mathbf{R}^{d}$ with unit diameter and $d \geq 3$.

Next, we require that the functional $\mathcal{F}$ satisfies some regularity properties. Namely,

(H2)

i. The functional $\mathcal{F}: L^{2}(\Omega, H) \rightarrow \mathbf{R}$ is differentiable. Denoting by $\mathbf{k}[\mathbf{p}] \in L^{2}(\Omega, H)$ the gradient of $\mathcal{F}$ at some point $\mathbf{p} \in L^{2}(\Omega, H)$ and by $\mathcal{B}$ the convex hull of $\mathcal{S}$ in $H$, we assume that there exists $C_{1} \geq 0$, such that

$$
\|\mathbf{k}[\mathbf{p}]\|_{L^{2}} \leq C_{1} \quad \text { for every } \mathbf{p} \in L^{2}(\Omega, \mathcal{B}) .
$$

ii. The mapping $\mathbf{p} \in L^{2}(\Omega, H) \mapsto \mathbf{k}[\mathbf{p}] \in L^{2}(\Omega, H)$ is Gâteaux differentiable and there exists $C_{2} \geq 0$ such that

$$
\|D \mathbf{k}[\mathbf{p}] \cdot \mathbf{q}\|_{L^{2}} \leq C_{2}\|\mathbf{q}\|_{L^{2}} \quad \text { for every } \mathbf{p} \in L^{2}(\Omega, \mathcal{B}), \mathbf{q} \in L^{2}(\Omega, H)
$$


iii. If $\mathbf{p} \in L^{2}(\Omega, H)$ and if $K$ is a compact subset of $L^{2}(\Omega, H)$, the convergence

holds uniformly in $\mathbf{q} \in K$.

$$
\left\|\frac{\mathbf{k}[\mathbf{p}+t \mathbf{q}]-\mathbf{k}[\mathbf{p}]}{t}-D \mathbf{k}[\mathbf{p}] \cdot \mathbf{q}\right\|_{L^{2}} \stackrel{t \downarrow 0}{\longrightarrow} 0 .
$$

iv. If $\mathbf{p} \in H^{1}(\Omega, \mathcal{B})$, then $\mathbf{k}[\mathbf{p}]$ belongs to $H^{1}(\Omega, H)$ and there exists $C_{3} \geq 0$ such that

$$
\|\nabla \mathbf{k}[\mathbf{p}]\|_{L^{2}} \leq C_{3}\left(1+\|\nabla \mathbf{p}\|_{L^{2}}\right) \quad \text { for every } \mathbf{p} \in H^{1}(\Omega, \mathcal{B}) .
$$

For the exact rigidity result, we require,

(H3) The gradient $\mathbf{k}=\nabla_{L^{2}} \mathcal{F}$ maps $U(\Omega, H)$ into itself. Moreover, there exists $C_{3}^{\prime} \geq 0$, such that

$$
\|\nabla \mathbf{k}[\mathbf{p}]\|_{L^{2}} \leq C_{3}^{\prime}\|\nabla \mathbf{p}\|_{L^{2}} \quad \text { for every } \mathbf{p} \in H^{1}(\Omega, H) .
$$

We also need $\mathcal{S}$ to be a smooth manifold with a large group of isometries.

$(\mathbf{H 4}) \mathcal{S}$ is a smooth manifold. Moreover,

i. There exists a constant $C_{\mathcal{S}} \geq 0$, such that for every $\sigma \in \mathcal{S}$ and $\zeta \in T_{\sigma} \mathcal{S}$ there exists a smooth one parameter group $\{R(t)\}_{t \in \mathbf{R}}$ of isometries of $\mathcal{S}$ such that $\dot{R}(0) \sigma=\zeta$ and $\|\dot{R}(0)\|_{\infty} \leq C_{\mathcal{S}}|\zeta|$.

ii. There exists $C_{\mathcal{S}}^{\prime} \geq 0$, such that

$$
\left|\left(\sigma^{\prime}-\sigma\right) \cdot \xi\right| \leq C_{\mathcal{S}}^{\prime}\left|\sigma^{\prime}-\sigma\right|^{2}|\xi| \quad \text { for every } \sigma, \sigma^{\prime} \in \mathcal{S}, \xi \in N_{\sigma} \mathcal{S},
$$

where $N_{\sigma} \mathcal{S}$ denotes the orthogonal space to $T_{\sigma} \mathcal{S}$ in $H$.

Theorem 14. Let $\Omega \subset \mathbf{R}^{d}$, let $\mathcal{S}$ be a closed subset of some Hilbert $H$, let $\varepsilon>0$ and assume that $\mathbf{m}$ is a local minimizer of $\mathcal{D}_{\varepsilon}:=\mathcal{D}+\varepsilon^{2} \mathcal{F}$ in $H^{1}(\Omega, \mathcal{S})$ then:

i. if hypotheses $(\boldsymbol{H} 1-\mathbf{H} 2)$ hold, there exists $C_{\mathcal{F}} \geq 0$ and $\varepsilon_{\mathcal{F}}>0$ such that

$$
\varepsilon<\varepsilon_{\mathcal{F}} \quad \Longrightarrow\|\nabla \mathbf{m}\|_{L^{2}}^{2} \leq C_{\mathcal{F}} \varepsilon^{2} .
$$

ii. if moreover, $\left(\boldsymbol{H} 3-H_{4}\right)$ hold then, there exists $\varepsilon_{\mathcal{F}}^{\prime}$ such that if $\varepsilon<\varepsilon_{\mathcal{F}}^{\prime}$ then $\mathbf{m}$ is constant in $\Omega$.

This result is established in Section 4.

Example 15. If $\Omega$ is bounded, Hypotheses (H2) are satisfied by functional $\mathcal{F}$ of the form

$$
\mathcal{F}(\mathbf{p})=F\left[\frac{1}{2} \int_{\Omega \times \Omega} A\left(x_{1}, x_{2}\right)\left(\mathbf{p}\left(x_{1}\right), \mathbf{p}\left(x_{2}\right)\right) d x_{1} d x_{2}+\int_{\Omega} \psi(x, \mathbf{p}) d x\right]
$$

with $F \in C^{2}(\mathbf{R}, \mathbf{R}), A \in C_{c}^{2}\left(\bar{\Omega}^{2}, S\right)$ where $S$ is the space of continuous symmetric bilinear forms on $H, A\left(x_{1}, x_{2}\right)$ is symmetric for almost every $\left(x_{1}, x_{2}\right)$, and $\psi \in C_{c}^{2}(\bar{\Omega} \times H)$. If moreover $\psi$ does not depend on $x$ and

$$
\nabla_{x} \int_{\Omega} A(x, z) d z \equiv 0 \text { in } \Omega \text {, for every } \sigma \in \mathcal{S},
$$

then $\mathcal{F}$ satisfies hypothesis (H3). This is precisely the situation of Theorem 8.ii. (with $F$ linear). Hypotheses (H3) and (H4) are satisfied for $\mathcal{S}=\mathbf{R}, \mathbb{T}^{N}, \mathbb{S}^{N-1}, \mathrm{SO}_{N}(\mathbf{R}), \mathbb{S}^{N-1} /\{-1,1\}$. More generally, if $H$ is a Hilbert space, these hypotheses are satisfied for example if

. $\mathcal{S}=H$,

- $\mathcal{S}$ is the sphere $\{\sigma \in H ;|\sigma|=1\}$,

- $\mathcal{S}=G \sigma$ where $G$ is a manifold of $O(H)$ which is also a subgroup and $\sigma \in H \backslash\{0\}$,

- $\mathcal{S}=H / G$ when $G$ is a discrete subgroup of $O(H)$. 


\subsection{Proof of Theorem 8 (regularity properties of the micromagnetic energy)}

We assume here that $p=2, d=3, \mathcal{S}=\mathbb{S}^{2} \subset \mathbf{R}^{3}=H$ and that $\Omega \subset \mathbf{R}^{3}$ is a $C^{2}$ uniformly convex domain with unit diameter (i.e. (H1) holds). We fix $\varepsilon>0$ and consider the perturbation of the Dirichlet energy $\mathcal{D}_{\varepsilon}=\mathcal{D}+\varepsilon^{2} \mathcal{F}$ introduced in (1). We assume that $\psi \in C^{2}\left(\mathbb{S}^{2}, \mathbf{R}\right)$ and that $\mathbf{m}$ is a local minimizer of $\mathcal{D}_{\varepsilon}$ in $H^{1}\left(\Omega, \mathbb{S}^{2}\right)$.

Let us check step by step that the hypotheses of Theorem 14 are satisfied. Writing

$$
\mathcal{F}=\mathcal{F}_{\mathrm{d}}+\mathcal{F}_{\text {loc }} \quad \text { with } \quad \mathcal{F}_{\mathrm{d}}(\mathbf{p}):=-\frac{1}{2} \int_{\Omega} \mathbf{h}_{d}[\mathbf{p} ; \Omega](x) \cdot \mathbf{p}(x) d x, \quad \mathcal{F}_{\mathrm{d}}(\mathbf{p}):=\int_{\Omega} \psi(\mathbf{p}(x)) d x,
$$

we remark that $\mathcal{F}$ can be extended to a functional on $L^{2}\left(\Omega, \mathbf{R}^{3}\right)$. Indeed, $\mathbf{p} \mapsto-\mathbf{h}_{d}[\mathbf{p} ; \Omega]$ is a continuous linear projector of $L^{2}\left(\Omega, \mathbf{R}^{3}\right)$ while setting $\psi(\sigma):=\rho(|\sigma|) \psi(\sigma /|\sigma|)$ where $\rho \in C_{c}^{\infty}(0,2)$ satisfies $\rho \equiv 1$ on some neighborhood of 1 makes $\psi \in C_{c}^{2}\left(\mathbf{R}^{3}\right)$ and $\mathcal{F}_{\text {loc }}$ well defined on $L^{2}\left(\Omega, \mathbf{R}^{3}\right)$.

The next propositions state that $\mathcal{F}_{\mathrm{d}}$ and $\mathcal{F}_{\text {loc }}$ comply to the requirements of Theorem 14 .

Proposition 16. The functional $\mathcal{F}_{\mathrm{d}}$ satisfies hypothesis (H2).

Proof. i.-ii.-iii. Since $\mathbf{p} \mapsto-\mathbf{h}_{d}[\mathbf{p} ; \Omega]$ is a linear projection on the closed subspace $V$ of $L^{2}\left(\Omega, \mathbf{R}^{3}\right), \mathcal{F}_{\mathrm{d}}$ is a continuous quadratic functional on $L^{2}\left(\Omega, \mathbf{R}^{3}\right)$, with norm bounded by $1 / 2$. Therefore it is infinitely continuously differentiable, the gradient of $\mathcal{F}_{\mathrm{d}}$ at some point $\mathbf{p} \in L^{2}\left(\Omega, \mathbf{R}^{3}\right)$ being given by $\mathbf{k}_{\mathrm{d}}[\mathbf{p}]=-\mathbf{h}_{d}[\mathbf{p} ; \Omega]$, while $\forall \mathbf{q} \in L^{2}\left(\Omega, \mathbf{R}^{3}\right)$ one has $D \mathbf{k}_{\mathrm{d}}[\mathbf{p}] \cdot \mathbf{q}=-\mathbf{h}_{d}[\mathbf{q} ; \Omega]$. We also have the bounds

$$
\left\|\mathbf{k}_{\mathrm{d}}[\mathbf{p}]\right\|_{L^{2}} \leq\|\mathbf{p}\|_{L^{2}}, \quad\left\|D \mathbf{k}_{\mathrm{d}}[\mathbf{p}] \cdot \mathbf{q}\right\|_{L^{2}} \leq\|\mathbf{q}\|_{L^{2}} .
$$

In particular, (H2)-i.-ii.-iii. hold with $C_{1}=\sqrt{|\Omega|}, C_{2}=1$.

$i v$. We have to check that $\mathbf{p} \mapsto \mathbf{k}_{d}[\mathbf{p}]=-\mathbf{h}_{d}[\mathbf{p}, \Omega]$ maps $H^{1}\left(\Omega, \mathbf{R}^{3}\right)$ into itself. For this, we invoke Proposition 17 below and conclude that $\mathcal{F}_{\mathrm{d}}$ satisfies (H2)-iv. with $C_{3}=\max (1, \sqrt{|\Omega|}) C_{3}^{\prime \prime}$.

Proposition 17. ([8] Lemma 2.3.) Let $\Omega$ be a bounded domain of class $C^{2}$. If $\mathbf{p} \in H^{1}\left(\Omega, \mathbf{R}^{3}\right)$, then the restriction of $\mathbf{h}_{d}[\mathbf{p}, \Omega]$ to $\Omega$ belongs to $H^{1}\left(\Omega, \mathbf{R}^{3}\right)$. Moreover there exists a constant $C_{3}^{\prime \prime}=C_{3}^{\prime \prime}(\Omega)$ such that

$$
\left\|\mathbf{h}_{\mathrm{d}}[\mathbf{p}]\right\|_{H^{1}(\Omega)} \leq C_{3}^{\prime \prime}\|\mathbf{p}\|_{H^{1}}, \quad \text { for every } \mathbf{p} \in H^{1}\left(\Omega, \mathbf{R}^{3}\right) .
$$

Proposition 18. The functional $\mathcal{F}_{\text {loc }}$ satisfies hypothesis (H2).

Proof. (H2)- $i$. For $\mathbf{p} \in L^{2}\left(\Omega, \mathbf{R}^{3}\right)$, since $\psi \in \mathcal{C}_{c}^{2}\left(\mathbf{R}^{3}\right)$, the gradient of $\mathcal{F}_{\text {loc }}$ at $\mathbf{p}$ is given by

$$
\mathbf{k}_{\mathrm{loc}}[\mathbf{p}](x):=\nabla \psi(\mathbf{p}(x)), \forall x \in \Omega .
$$

We notice that the operator $\mathbf{p} \in L^{2}\left(\Omega, \mathbf{R}^{3}\right) \mapsto \mathbf{k}_{\text {loc }}[\mathbf{p}] \in L^{2}\left(\Omega, \mathbf{R}^{3}\right)$ is continuous, with

$$
\left\|\mathbf{k}_{\mathrm{loc}}[\mathbf{p}]\right\|_{L^{2}} \leq\|\nabla \psi\|_{\infty} \sqrt{|\Omega|} .
$$

Thus $\mathcal{F}_{\text {loc }}$ satisfies (H2)- $i$. with constant $C_{1}=\sqrt{|\Omega|}\|\nabla \psi\|_{\infty}$.

(H2)-ii. Now, since $\psi \in \mathcal{C}^{2}, \mathbf{p} \in L^{2}\left(\Omega, \mathbf{R}^{3}\right) \mapsto \mathbf{k}_{\text {loc }}[\mathbf{p}] \in L^{2}\left(\Omega, \mathbf{R}^{3}\right)$, is Gâteaux differentiable and one has $D \mathbf{k}_{\text {loc }}[\mathbf{p}] \cdot \mathbf{q}=D^{2} \psi(\mathbf{p}) \cdot \mathbf{q}$, for $\mathbf{p}, \mathbf{q} \in L^{2}\left(\Omega, \mathbf{R}^{3}\right)$. Therefore, $\mathcal{F}_{\text {loc }}$ satisfies (H2)-ii. with $C_{2}=\left\|D^{2} \psi\right\|_{\infty}$.

(H2)-iii. Let $\mathbf{p} \in L^{2}\left(\Omega, \mathbf{R}^{3}\right)$ and let $K$ be a compact set of $L^{2}\left(\Omega, \mathbf{R}^{3}\right)$. For $\mathbf{q} \in K$ and $x \in \Omega$ and $t>0$, we set

$$
R(x ; \mathbf{q}, t):=\frac{\nabla \psi[\mathbf{p}(x)+t \mathbf{q}(x)]-\nabla \psi[\mathbf{p}(x)]}{t}-D^{2} \psi[\mathbf{p}(x)] \cdot \mathbf{q}(x),
$$

and we remark that, using the Taylor-Lagrange formula, we have

$$
R(x ; \mathbf{q}, t)=\left[D^{2} \psi\{\mathbf{p}(x)+t \zeta(x, t) \mathbf{q}(x)\}-D^{2} \psi\{\mathbf{p}(x)\}\right] \cdot \mathbf{q}(x),
$$


where $\zeta(x, t) \in(0,1)$. Let us introduce a parameter $\eta>0$ and split $\Omega$ into $\Omega_{\eta}:=\{x:|\mathbf{q}(x)|<\eta / t\}$ and $\Omega \backslash \Omega_{\eta}$. We have the obvious bounds

$$
\forall x \in \Omega \backslash \Omega_{\eta},|R(x ; \mathbf{q}, t)| \leq 2\left\|D^{2} \psi\right\|_{\infty}|\mathbf{q}(x)|,
$$

while

$$
\forall x \in \Omega_{\eta},|R(x ; \mathbf{q}, t)| \leq \omega(\eta)|\mathbf{q}(x)|,
$$

where $\omega$ is a modulus of continuity for $D^{2} \psi$ (recall that $\psi$ is of class $C^{2}$ and compactly supported). This leads to

$$
\int_{\Omega}|R(x ; \mathbf{q}, t)|^{2} d x \leq \omega^{2}(\eta)\|\mathbf{q}\|_{L^{2}}^{2}+4\left\|D^{2} \psi\right\|_{\infty}^{2} \int_{\{x:|\mathbf{q}(x)| \geq \eta / t\}}|\mathbf{q}(x)|^{2} d x .
$$

Since $K$ is compact in $L^{2}\left(\Omega, \mathbf{R}^{3}\right), K$ is bounded in $L^{2}\left(\Omega, \mathbf{R}^{3}\right)$

$$
\exists C_{K}>0, \forall \mathbf{q} \in K,\|\mathbf{q}\|_{L^{2}} \leq C_{K},
$$

and the functions $\left\{x \in \Omega \mapsto|\mathbf{q}(x)|^{2}: \mathbf{q} \in K\right\}$ are uniformly equi-integrable. Therefore for $\eta>0$ fixed the integral in the right hand side of (5) goes to 0 as $t \downarrow 0$ uniformly in $\mathbf{q} \in K$. This leads to

$$
\varlimsup_{t \downarrow 0}\left\{\sup _{\mathbf{q} \in K} \int_{\Omega}|R(x ; \mathbf{q}, t)|^{2} d x\right\} \leq C_{K} \omega^{2}(\eta) .
$$

Eventually, since $\eta$ is arbitrary and $\omega(\eta) \rightarrow 0$ as $\eta \downarrow 0$, the above limit vanishes, as required.

(H2)- $i v$. Let us now assume $\mathbf{p} \in H^{1}\left(\Omega, \mathbf{R}^{3}\right)$. We have to check that $\mathbf{k}[\mathbf{p}]$ belongs to $H^{1}\left(\Omega, \mathbf{R}^{3}\right)$. First, since $\psi \in C_{c}^{2}\left(\mathbf{R}^{3}\right)$, the mapping $x \mapsto \mathbf{k}_{\text {loc }}[\mathbf{p}](x)=\nabla \psi(\mathbf{p}(x))$ belongs to $H^{1}\left(\Omega, \mathbf{R}^{3}\right)$ and using the chain rule, we have the estimate,

$$
\left\|\nabla\left\{\mathbf{k}_{\mathrm{loc}}[\mathbf{p}]\right\}\right\|_{L^{2}} \leq\left\|D^{2} \psi\right\|_{\infty}\|\nabla \mathbf{p}\|_{L^{2}}
$$

We deduce from (6) that $\mathcal{F}_{\text {loc }}$ satisfies (H2)-iv. with $C_{3}=\left\|D^{2} \psi\right\|_{\infty}$.

For the second part of Theorem 8, we invoke Proposition 3 that states that, in solid ellipsoids, uniform magnetizations create uniform stray fields. Taking into account the (homogeneous) anisotropy, we have the following result.

Proposition 19. If $\Omega$ is a solid ellipsoid, then (H3) holds, that is to say $\mathbf{k}=\nabla_{L^{2}} \mathcal{F}$ maps $U\left(\Omega, \mathbf{R}^{3}\right)$ into itself and moreover, there exists $C_{3}^{\prime} \geq 0$, such that

$$
\|\nabla \mathbf{k}[\mathbf{p}]\|_{L^{2}} \leq C_{3}^{\prime}\|\nabla \mathbf{p}\|_{L^{2}} \quad \text { for every } \mathbf{p} \in H^{1}\left(\Omega, \mathbf{R}^{3}\right) .
$$

Proof. Let $\mathbf{u} \equiv \sigma \in U\left(\Omega, \mathbb{S}^{2}\right)$, then

$$
\mathbf{k}[\mathbf{u}](x)=-\mathbf{h}_{d}[\mathbf{u} ; \Omega](x)+\nabla \psi(\sigma) \quad \text { for every } x \in \Omega .
$$

By Proposition 3, we know that $\mathbf{h}_{d}[\mathbf{u} ; \Omega]$ is uniform inside $\Omega$. Thus $\mathbf{k}[\mathbf{u}] \in U\left(\Omega, \mathbb{S}^{2}\right)$.

Let us now establish the estimate. With the notation of the proof of Propositions 16 and 18, we have $\nabla \mathbf{k}[\mathbf{p}]=\nabla\left\{\mathbf{k}_{\text {loc }}[\mathbf{p}]\right\}-\nabla\left\{\mathbf{h}_{d}[\mathbf{p}]\right\}$, for every $\mathbf{p} \in H^{1}\left(\Omega, \mathbf{R}^{3}\right)$. For the first term, we have already established the desired estimate in $(6)$.

Next, let $\mathbf{p} \in H^{1}\left(\Omega, \mathbf{R}^{3}\right)$. By linearity of $\mathbf{p} \mapsto \mathbf{h}_{d}[\mathbf{p}]$, we have $\mathbf{h}_{d}[\mathbf{p}]=\mathbf{h}_{d}[\langle\mathbf{p}\rangle]+\mathbf{h}_{d}[\mathbf{p}-\langle\mathbf{p}\rangle]$. Since $\Omega$ is a solid ellipsoid, it follows from Proposition 3 that $\mathbf{h}_{d}[\langle\mathbf{p}\rangle]$ is constant in $\Omega$. Hence $\nabla\left\{\mathbf{h}_{d}[\mathbf{p}]\right\}=\nabla\left\{\mathbf{h}_{d}[\mathbf{p}-\langle\mathbf{p}\rangle]\right\}$. Using Proposition 17 and the Poincaré inequality (2), we get

$$
\left\|\nabla\left\{\mathbf{h}_{d}[\mathbf{p}]\right\}\right\|_{L^{2}}^{2} \leq\left(C_{3}^{\prime \prime}\right)^{2}\left(\|\mathbf{p}-\langle\mathbf{p}\rangle\|_{L^{2}}^{2}+\|\nabla \mathbf{p}\|_{L^{2}}^{2}\right) \leq\left(C_{3}^{\prime \prime}\right)^{2}\left(1+C_{P}^{2}\right)\|\nabla \mathbf{p}\|_{L^{2}}^{2} .
$$

This establishes the estimate of (H3) with $C_{3}^{\prime}=\left\|D^{2} \psi\right\|_{\infty}+C_{3}^{\prime \prime} \sqrt{1+C_{P}^{2}}$.

Eventually, we check that $\mathbb{S}^{2} \subset \mathbf{R}^{3}$ satisfies (H4).

Lemma 20. The sphere $\mathcal{S}=\mathbb{S}^{2}$ satisfies (H4). 
Proof. $i$. Let $\sigma \in \mathbb{S}^{2}, \zeta \in \sigma^{\perp}=T_{\sigma} \mathbb{S}^{2}$ and call $\zeta^{\prime}:=\sigma \times \zeta$. Let us define the one parameter group of rotations $R(t)=e^{t A}$ where $A$ is the skew symmetric matrix given by $A \sigma^{\prime}=\zeta^{\prime} \times \sigma^{\prime}$. This group satisfies $\dot{R}(0) \sigma=A \sigma=\zeta$ and the estimate $\|\dot{R}(0)\|_{\infty} \leq C_{\mathbb{S}^{2}}|\zeta|$ holds with $C_{\mathbb{S}^{2}}=1$.

ii. For $\sigma \in \mathbb{S}^{2}$, we have $N_{\sigma} \mathcal{S}=\mathbf{R} \sigma$, so we may assume $\xi=\lambda \sigma$. We compute for $\sigma^{\prime} \in \mathbb{S}^{2}$,

$$
\left(\sigma-\sigma^{\prime}\right) \cdot \xi=\lambda\left(1-\sigma^{\prime} \cdot \sigma\right)=\frac{\lambda}{2}\left|\sigma^{\prime}-\sigma\right|^{2} .
$$

Hence, (H4)-ii. holds with $C_{\mathbb{S}^{2}}^{\prime}=1 / 2$.

As a conclusion, by Propositions 16 and 18, Theorem 8.i. is a consequence of Theorem 14.i. and then by Proposition 19 and Lemma 20, Theorem 8.ii. follows from Theorem 14.ii.

\subsection{Proof of Theorem 5}

At this point, we have at hand all the tools to prove Theorem 5 .

Proof. (of Theorem 5) Let $\Omega$ be an ellipsoid, assume that $\psi$ is of class $C^{2}$ and let $\mathbf{m}$ minimizing $\mathcal{D}_{\varepsilon}$ in $H^{1}\left(\Omega, \mathbb{S}^{2}\right)$. We denote by $\langle\mathbf{m}\rangle=\frac{1}{|\Omega|} \int_{\Omega} \mathbf{m}(x) d x$ the average value of $\mathbf{m}$ on $\Omega$ and define

$$
\sigma=\left\{\begin{array}{c}
\frac{\langle\mathbf{m}\rangle}{|\langle\mathbf{m}\rangle|} \text { if }\langle\mathbf{m}\rangle \neq 0, \\
\text { any point in } \mathbb{S}^{2} \text { otherwise. }
\end{array}\right.
$$

Eventually, let $\mathbf{u} \equiv \sigma \in U\left(\Omega, \mathbb{S}^{2}\right)$. By optimality of $\mathbf{m}$, we have, $\mathcal{D}_{\varepsilon}(\mathbf{m}) \leq \mathcal{D}_{\varepsilon}(\mathbf{u})=\varepsilon^{2} \mathcal{F}(\mathbf{u})$, thus

$$
\|\nabla \mathbf{m}\|_{L^{2}}^{2} \leq 2 \varepsilon^{2}[\mathcal{F}(\mathbf{u})-\mathcal{F}(\mathbf{m})]
$$

Next, by Proposition 16. and Proposition 18, $\mathcal{F}$ is differentiable in $L^{2}\left(\Omega, \mathbf{R}^{3}\right)$ and its gradient is given by $\mathbf{k}[\mathbf{p}](x)=-\mathbf{h}_{d}[\mathbf{p} ; \Omega](x)+\nabla \psi(\mathbf{p}(x))$. So,

$$
\mathcal{F}(\mathbf{u})-\mathcal{F}(\mathbf{m})=-\int_{0}^{1} \int_{\Omega} \mathbf{k}[\mathbf{u}+t(\mathbf{m}-\mathbf{u})](x) \cdot[\mathbf{m}-\mathbf{u}](x) d x d t .
$$

Since $\mathbf{k}[\mathbf{u}]$ does not depend on $x \in \Omega$, we obtain

$$
\mathcal{F}(\mathbf{u})-\mathcal{F}(\mathbf{m})=-\mathbf{k}[\mathbf{u}] \cdot \int_{\Omega}[\mathbf{m}-\mathbf{u}]+\int_{0}^{1} \int_{\Omega}\{\mathbf{k}[\mathbf{u}]-\mathbf{k}[\mathbf{u}+t(\mathbf{m}-\mathbf{u})]\} \cdot[\mathbf{m}-\mathbf{u}] d t .
$$

From the expression of $\mathbf{k}$, this leads to

$$
\mathcal{F}(\mathbf{u})-\mathcal{F}(\mathbf{m}) \leq\left(1+\|\nabla \psi\|_{\infty}\right)\left|\int_{\Omega}[\mathbf{m}-\mathbf{u}]\right|+\frac{1}{2}\left(1+\left\|D^{2} \psi\right\|_{\infty}\right)\|\mathbf{m}-\mathbf{u}\|_{L^{2}}^{2}
$$

We first bound the integral in the right hand side. By definition of $\mathbf{u}$,

$$
\int_{\Omega}[\mathbf{m}-\mathbf{u}]=|\Omega|(\langle\mathbf{m}\rangle-\sigma)=|\Omega|(|\langle\mathbf{m}\rangle|-1) \sigma .
$$

On the other hand, since $|\mathbf{m}(x)|=1$ a.e. in $\Omega$, we have

$$
\int_{\Omega}|\mathbf{m}-\langle\mathbf{m}\rangle|^{2}=|\Omega|\left(1-|\langle\mathbf{m}\rangle|^{2}\right) .
$$

Using $|\langle\mathbf{m}\rangle| \leq 1$ and Poincaré inequality, we obtain

$$
\left|\int_{\Omega}[\mathbf{m}-\mathbf{u}]\right|=|\Omega|(1-|\langle\mathbf{m}\rangle|) \leq|\Omega|\left(1-|\langle\mathbf{m}\rangle|^{2}\right) \leq \int_{\Omega}|\mathbf{m}-\langle\mathbf{m}\rangle|^{2} \leq C_{P}^{2}\|\nabla \mathbf{m}\|_{L^{2}}^{2} .
$$


Next, we bound the last term $\|\mathbf{m}-\mathbf{u}\|_{L^{2}}^{2}$. Since $\mathbf{m} \in L^{2}\left(\Omega, \mathbb{S}^{2}\right)$ and $\mathbf{u}$ minimizes the distance $|\mathbf{v}-\langle\mathbf{m}\rangle|$ in $\mathbb{S}^{2}$, we have $|\mathbf{u}-\langle\mathbf{m}\rangle| \leq|\mathbf{m}(x)-\langle\mathbf{m}\rangle|$ for almost every $x \in \Omega$ which gives the bound $\|\mathbf{u}-\langle\mathbf{m}\rangle\|_{L^{2}}^{2} \leq$ $\|\mathbf{m}-\langle\mathbf{m}\rangle\|_{L^{2}}^{2}$. Therefore

$$
\|\mathbf{m}-\mathbf{u}\|_{L^{2}} \leq\|\mathbf{m}-\langle\mathbf{m}\rangle\|_{L^{2}}+\|\langle\mathbf{m}\rangle-\mathbf{u}\|_{L^{2}} \leq 2\|\mathbf{m}-\langle\mathbf{m}\rangle\|_{L^{2}} \leq 2 C_{P}\|\nabla \mathbf{m}\|_{L^{2}}
$$

from Poincaré inequality. Eventually, we have obtained,

$$
\mathcal{F}(\mathbf{u})-\mathcal{F}(\mathbf{m}) \leq C_{P}^{2}\left(3+\|\nabla \psi\|_{\infty}+2\left\|D^{2} \psi\right\|_{\infty}\right)\|\nabla \mathbf{m}\|_{L^{2}}^{2} .
$$

Together with (7), we get that if $\varepsilon^{2}<1 / 2 C_{P}^{2}\left(3+\|\nabla \psi\|_{\infty}+2\left\|D^{2} \psi\right\|_{\infty}\right)$, then $\mathbf{m}$ is constant.

\section{Proof of Theorem 9}

Throughout this Section, we assume that $\Omega \subset \mathbf{R}^{d}$ is a bounded open set which is star-shaped with respect to some point $x_{0} \in \mathbf{R}^{d}$. Without loss of generality, we assume $x_{0}=0$. We let $p>1$ and $\mathcal{S}$ be a closed subset of some Hilbert space $H$. Eventually we assume that $\mathbf{m} \in W^{1, p}(\Omega, \mathcal{S})$ is a local minimizer of $\mathcal{E}$ in this set for the $W^{1, p}$-topology.

In order to prove Theorem 9 , we compare the energy of $\mathbf{m}$ with some competitors $\left\{\mathbf{m}_{t}\right\}_{t \in\left(0, t_{0}\right)} \subset W^{1, p}(\Omega$, $\mathcal{S})$. As already noticed, the usual perturbations of the form $\mathbf{m}_{t}=\mathbf{m}+t \varphi$ are not allowed and we use instead the so-called inner variations

$$
\mathbf{m}_{t}=\mathbf{m} \circ(\operatorname{Id}+t \varphi)
$$

for some suitable $\varphi \in C^{\infty}\left(\bar{\Omega}, \mathbf{R}^{d}\right)$. Notice that $\varphi$ is not supposed to vanish on $\partial \Omega$ and hence we have the following two possibilities.

- If $(\operatorname{Id}+t \varphi)(\Omega) \subset \Omega$ for $t>0$ small enough, $\operatorname{Id}+t \varphi$ is a diffeomorphism from $\Omega$ onto a open subset of $\Omega, \mathbf{m}_{t}$ is well defined and $\mathbf{m}_{t} \in W^{1, p}(\Omega, H)$ since by the chain rule,

$$
\partial_{i} \mathbf{m}_{t}(x)=\left[\partial_{i} \mathbf{m}+t \partial_{i} \varphi(x) \cdot \nabla \mathbf{m}\right](x+t \varphi(x)) \quad \text { for } i=1, \cdots, d \text { and for a.e. } x \in \Omega .
$$

We also have $\mathbf{m}_{t}(x) \in \mathcal{S}$ almost everywhere in $\Omega$, so that $\mathbf{m}_{t} \in W^{1, p}(\Omega, \mathcal{S})$.

- If $(\operatorname{Id}+t \varphi)(\Omega) \not \subset \Omega$, then we first have to consider a extension $\tilde{\mathbf{m}} \in W^{1, p}(\mathcal{O}, \mathcal{S})$ defined on some open neighborhood $\mathcal{O}$ of $\bar{\Omega}$ and such that $\tilde{\mathbf{m}}_{\mid \Omega}=\mathbf{m}$. We then set $\mathbf{m}_{t}=\tilde{\mathbf{m}} \circ(\operatorname{Id}+t \varphi)$.

In both cases, we have $\mathbf{m}_{t} \rightarrow \mathbf{m}$ as $t \downarrow 0$ in $W^{1, p}(\Omega, H)$ (we can see this by using the density of $C^{\infty}(\mathcal{O}, H)$ in $\left.W_{\text {loc }}^{1, p}(\mathcal{O}, H)\right)$, and hence, by local optimality of $\mathbf{m}$, there exists $\eta=\eta(\tilde{\mathbf{m}}, \varphi)$ such that

$$
0<t<\eta \quad \Longrightarrow \quad \mathcal{E}(\mathbf{m}) \leq \mathcal{E}\left(\mathbf{m}_{t}\right) \text {. }
$$

In what follows, we consider a family of such inner variations $\left\{\left\{\mathbf{m}_{t}^{\theta}:=\tilde{\mathbf{m}} \circ\left(\operatorname{Id}+t \varphi^{\theta}\right)\right\}_{t}\right\}_{\theta \in J}$ where $\left\{\varphi^{\theta}\right\}_{\theta \in J} \subset C^{\infty}\left(\bar{\Omega}, \mathbf{R}^{d}\right)$. We need the following Lemma.

Lemma 21. If $\left\{\varphi^{\theta}\right\}_{\theta \in J}$ is compact in $C^{1}\left(\bar{\Omega}, \mathbf{R}^{d}\right)$ then the convergence $\mathbf{m}_{t}^{\theta} \stackrel{t \downarrow 0}{\longrightarrow} \mathbf{m}$ in $W^{1, p}(\Omega, H)$ is uniform in $\theta \in J$.

Proof. Denoting by $L_{\theta}$ the Lipschitz constant of $\varphi^{\theta}$, we set

$$
\eta:=\inf _{\theta \in J} \min \left(\frac{d\left(\Omega, \mathcal{O}^{c}\right)}{\left\|\varphi^{\theta}\right\|_{\infty}}, \frac{1}{L_{\theta}}\right)>0 .
$$

Then, as soon as $0<t<\eta, \operatorname{Id}+t \varphi^{\theta}$ is a diffeomorphism of $\Omega$ onto a relatively compact subset of $\mathcal{O}$ and $\mathbf{m}_{t}^{\theta}$ is a well defined element of $W^{1, p}(\Omega, \mathcal{S})$ for every $\theta \in J$. Now, assume by contradiction, that there exist $\delta>0$ and two sequences $\left(\theta_{k}\right)_{k} \subset J$ and $\left(t_{k}\right)_{k}$ such that $t_{k} \downarrow 0$ and

$$
\left\|\mathbf{m}_{t_{k}}^{\theta_{k}}-\mathbf{m}\right\|_{W^{1, p}}>\delta \quad \text { for every } k \geq 0 .
$$


Up to extraction, we may assume that $\left(\varphi^{\theta_{k}}\right)_{k}$ converges towards $\varphi^{\theta}$ in $C^{1}\left(\bar{\Omega}, \mathbf{R}^{d}\right)$, and therefore $\left(\operatorname{Id}+t_{k} \varphi^{\theta_{k}}\right)_{k}$ converges to Id in $C^{1}\left(\bar{\Omega}, \mathbf{R}^{d}\right)$. We thus see that $\left(\left|\mathbf{m}_{t_{k}}^{\theta_{k}}\right|^{p}+\left|\nabla \mathbf{m}_{t_{k}}^{\theta_{k}}\right|^{p}\right)_{k}$ is uniformly equi-integrable in $\Omega$. When $H$ is a finite dimensional space, this is sufficient to conclude that $\left(\mathbf{m}_{t_{k}}^{\theta_{k}}\right)$ is relatively compact in $W^{1, p}(\Omega, H)$. We then have, up to extraction $\mathbf{m}_{t_{k}}^{\theta_{k}} \rightarrow \mathbf{w} \in W^{1, p}(\Omega, H)$. But we also have $\mathbf{m}_{t_{k}}^{\theta_{k}} \rightarrow \mathbf{m}$ in $\mathcal{D}^{\prime}(\Omega, H)$, so that we can identify $\mathbf{w}=\mathbf{m}$ contradicting (11).

When $H$ is not finite dimensional, we need another argument. First we notice that we can approximate $\mathbf{m}$ in $L^{1}(\Omega, H)$ by a sequence $\left(\mathbf{s}_{j}\right)_{j}$ of simple measurable functions (the range of $\mathbf{s}_{j}$ is a finite set $A_{j} \subset H$ ). We then set $H_{j}:=\operatorname{span}\left\{\cup_{l \leq j} A_{l}\right\}$ and call $P_{j}$ the orthogonal projector on $H_{j}$. Since this projection is a contraction, we have

$$
\left|P_{j} \mathbf{m}\right|(x) \leq|\mathbf{m}|(x) \quad \text { and } \quad\left|\nabla P_{j} \mathbf{m}\right|(x) \leq|\nabla \mathbf{m}|(x) \quad \text { a.e. in } \Omega .
$$

On the other hand $P_{j} \mathbf{m}(x) \rightarrow \mathbf{m}(x)$ and for $1 \leq k \leq d, \quad \partial_{k} P_{j} \mathbf{m}(x)=P_{j} \partial_{k} \mathbf{m} \rightarrow \partial_{k} \mathbf{m}(x)$ almost everywhere in $\Omega$, therefore by Lebesgue's dominated convergence Theorem, we have $P_{j} \mathbf{m} \rightarrow \mathbf{m}$ in $W^{1, p}(\Omega, H)$.

For any $\delta>0$, we fix $j$ such that $\left\|P_{j} \mathbf{m}-\mathbf{m}\right\|_{W^{1, p}}<\delta / 3$. A direct computation using (10) and the change of variable $y=x+t_{k} \varphi^{\theta_{k}}(x)$ shows that $\left\|P_{j} \mathbf{m}_{t_{k}}^{\theta_{k}}-\mathbf{m}_{t_{k}}^{\theta_{k}}\right\|_{W^{1, p}} \leq\left(1+O\left(t_{k}\right)\right)\left\|P_{j} \tilde{\mathbf{m}}-\tilde{\mathbf{m}}\right\|_{W^{1, p}\left(\Omega+B\left(0, t_{k}\left\|\varphi^{\theta_{k}}\right\|_{\infty}\right)\right)}$, so we also have $\left\|P_{j} \mathbf{m}_{t_{k}}^{\theta_{k}}-\mathbf{m}_{t_{k}}^{\theta_{k}}\right\|_{W^{1, p}}<\delta / 3$ for $k$ large enough.

Eventually, by the definition of $P_{j}$, we have $P_{j} \mathbf{m}_{t_{k}}^{\theta_{k}}=\left(P_{j} \mathbf{m}\right) \circ\left(\operatorname{Id}+t_{k} \varphi^{\theta_{k}}\right)$ and from the finite dimensional case, we know that for $k$ large enough $\left\|P_{j} \mathbf{m}_{t_{k}}^{\theta_{k}}-P_{j} \mathbf{m}\right\|_{W^{1, p}}<\delta / 3$. These estimates yield the desired contradiction

$$
\left\|\mathbf{m}_{t_{k}}^{\theta_{k}}-\mathbf{m}\right\|_{W^{1, p}} \leq\left\|P_{j} \mathbf{m}_{t_{k}}^{\theta_{k}}-\mathbf{m}_{t_{k}}^{\theta_{k}}\right\|_{W^{1, p}}+\left\|P_{j} \mathbf{m}_{t_{k}}^{\theta_{k}}-P_{j} \mathbf{m}\right\|_{W^{1, p}}+\left\|P_{j} \mathbf{m}-\mathbf{m}\right\|_{W^{1, p}}<\delta
$$

for $k$ large enough.

In the sequel, we only use three kinds of inner variations. In Section 3.1, we first consider dilations with coefficient $(1-t)$, which amounts to choose $\varphi(x)=-x$ in (9). Since $\Omega$ is star-shaped with respect to 0 , there is no need to extend $\mathbf{m}$ outside $\Omega$ in that case. This turns out to be sufficient to establish parts $i$. and $i$. of Theorem 9 . Then, assuming that $\Omega$ is $C^{2}$ and convex, we introduce a particular extension of $\mathbf{m}$ and consider dilations with coefficient $(1+t)$ which correspond to the choice $\varphi(x)=x$ in $(9)$. In these two steps, we do not really need $\mathbf{m}$ to be a local minimizer, we only use the weaker first order condition

$$
\mathcal{E}\left(\mathbf{m}_{t}\right) \geq \mathcal{E}(\mathbf{m})+o(t) .
$$

In Section 3.2, we consider translations of the domain, that is to say inner variations generated by the family of perturbations $\left\{\varphi^{\theta}(x)=\theta\right\}_{\theta \in \mathbb{S}^{d-1}}$. For this step, we make use of the second order optimality condition

$$
\mathcal{E}\left(\mathbf{m}_{t}^{\theta}\right) \geq \mathcal{E}(\mathbf{m})+o\left(t^{2}\right) .
$$

Since $\mathbb{S}^{d-1}$ is compact, by Lemma 21 , this optimality condition is satisfied uniformly in $\theta \in \mathbb{S}^{d-1}$ which is required for our proof. This is the reason why we ask for $\mathbf{m}$ to be a local minimizer for the $W^{1, p}$-topology (see the discussion in Section 5).

\subsection{Domain dilations (proof of parts $i, i i$ of Theorem 9 and preliminaries for $i i i$ )}

Lemma 22. (Zooming in (a)) Under the hypotheses of Theorem 9, we have for $t \in(0,1)$ small enough,

$$
\frac{\mathcal{E}(\mathbf{m} ; \Omega \backslash(1-t) \Omega)}{t} \leq \frac{1-(1-t)^{d-p}}{t} \mathcal{E}(\mathbf{m} ; \Omega) .
$$

Proof. We introduce a first family of inner variations of $\mathbf{m}$, namely for $t \in(0,1)$ and $x \in \Omega$, we set,

$$
\mathbf{m}_{t}(x):=\mathbf{m}((1-t) x) .
$$


Let us compare $\mathcal{E}\left(\mathbf{m}_{t}\right)$ and $\mathcal{E}(\mathbf{m})$. First, we compute $\nabla \mathbf{m}_{t}(x)=(1-t) \nabla \mathbf{m}((1-t) x)$. This identity holds in $L^{p}(\Omega)$, as soon as $t \in(0,1)$ and by the change of variable $y=(1-t) x$, we get for $t \in(0,1)$,

$$
\mathcal{E}\left(\mathbf{m}_{t} ; \Omega\right)=(1-t)^{p-d} \mathcal{E}(\mathbf{m} ;(1-t) \Omega) .
$$

By hypothesis, $\mathcal{E}(\mathbf{m} ; \Omega) \leq \mathcal{E}\left(\mathbf{m}_{t} ; \Omega\right)$ for $t$ small enough. Multiplying both sides of this inequality by $t^{-1}(1-$ $t)^{d-p}$ and simplifying, we obtain (12).

Let us notice that if $d<p$, then the coefficient in the right hand side is negative and since the left hand side is non negative, this leads to $\mathcal{E}(\mathbf{m} ; \Omega)=0$ and so $\mathbf{m}$ is constant in $\Omega$. This proves Theorem 9.i.

When $p=d$, we get $\mathcal{E}(\mathbf{m} ; \Omega \backslash(1-t) \Omega)=0$, which means that $\nabla \mathbf{m}$ is supported in $(1-t) \bar{\Omega}$. This implies part ii. of the Theorem.

From now on, we assume $d>p$ and that $\Omega$ has Lipschitz regularity and is star-shaped with respect to some non-empty open ball $B_{\rho}(0)$.

Lemma 23. (Zooming in (b)) The trace $\mathbf{u}_{0}$ of $\mathbf{m}$ on $\partial \Omega$ belongs to $W^{1, p}(\partial \Omega, \mathcal{S})$ and satisfies the estimates,

$$
\frac{1}{p} \int_{\partial \Omega}\left|\nabla \mathbf{u}_{0}\right|^{p}(y)(y \cdot \mathbf{n}(y)) d \mathcal{H}^{d-1}(y) \leq \lim _{t \downarrow 0} \frac{\mathcal{E}\left(\mathbf{m} ; \mathcal{M}_{t}^{-}\right)}{t} \leq \varlimsup_{t \downarrow 0} \frac{\mathcal{E}\left(\mathbf{m} ; \mathcal{M}_{t}^{-}\right)}{t} \leq(d-p) \mathcal{E}(\mathbf{m}),
$$

with $\mathcal{M}_{t}^{-}:=\Omega \backslash(1-t) \bar{\Omega}$, for $t \in(0,1)$.

Proof. The central inequality of (13) is obvious. Moreover, starting from (12) and taking the limsup as $t \downarrow 0$, we get the last inequality of (13).

We now study the left hand side of (12) and establish the first inequality of (13). Let us first notice that by the trace Theorem (and since $\Omega$ is star-shaped with respect to $B_{\rho}(0)$ ), $\mathbf{m}$ admits a representative defined on $\bar{\Omega}$, still denoted by $\mathbf{m}$, such that, if we set $\mathbf{u}_{t}(y)=\mathbf{m}((1-t) y)$ for $y \in \partial \Omega$ and $t \in[0,1)$, then $t \in[0,1) \mapsto \mathbf{u}_{t} \in L^{1}(\partial \Omega, \mathcal{S})$ is continuous. By definition, the trace of $\mathbf{m}$ on $\partial \Omega$ is $\mathbf{u}_{0}$. Since $\mathcal{S}$ is closed, we have $\mathbf{u}_{t} \in L^{1}(\partial \Omega, \mathcal{S})$, for every $t \in[0,1)$. In particular,

$$
\lim _{t \downarrow 0} \mathbf{u}_{t}=\mathbf{u}_{0} \quad \text { in } L^{1}(\partial \Omega, H) .
$$

We now introduce the family of change of variables

$$
\psi_{t}: \partial \Omega \times(0,1) \rightarrow \mathbf{R}^{d}, \quad(y, s) \mapsto(1-s t) y .
$$

For $t \in(0,1)$, the map $\psi_{t}$ defines a (bi-Lipschitz) diffeomorphism from $\partial \Omega \times(0,1)$ onto its image $\mathcal{M}_{t}^{-}$. Thus, we can define a family $\mathbf{v}_{t} \in H^{1}(\partial \Omega \times(0,1), \mathcal{S})$ by $\mathbf{v}_{t}(y, s):=\mathbf{m}\left(\psi_{t}(y, s)\right)$. Due to (14), we have

$$
\lim _{t \downarrow 0} \mathbf{v}_{t}=\mathbf{u}_{0} \quad \text { in } L^{1}(\partial \Omega \times(0,1), H),
$$

with the abuse of notation $\mathbf{u}_{0}(y, s)=\mathbf{u}_{0}(y)$.

Next, we show that $\nabla \mathbf{u}_{0} \in L^{p}\left(\partial \Omega, H^{d}\right)$. By the chain rule, the gradient of $\mathbf{v}_{t}$ with respect to any tangential direction $\xi \in T_{y} \partial \Omega$ reads

$$
\left(\xi \cdot \nabla_{y}\right) \mathbf{v}_{t}(y, s)=(1-s t)(\xi \cdot \nabla) \mathbf{m}\left(\psi_{t}(y, s)\right) .
$$

We write $\nabla_{y} \mathbf{v}_{t}(y, s)=(1-s t) \nabla_{\tau} \mathbf{m}\left(\psi_{t}(y, s)\right)$, where, for $x=\psi_{t}(y, s) \in \hat{\Omega}_{t}, \nabla_{\tau} \mathbf{m}(x)$ denotes the projection of $\nabla \mathbf{m}(x)$ onto $T_{y} \partial \Omega \times H \subset \mathbf{R}^{d} \times H$. The change of variable formula leads to

$$
\frac{1}{t p} \int_{\partial \Omega \times(0,1)}\left|\nabla_{y} \mathbf{v}_{t}\right|^{p}(y, s)(1-s t)^{-p} J_{t}(y, s) d s d \mathcal{H}^{d-1}(y)=\frac{1}{t p} \int_{\mathcal{M}_{t}^{-}}\left|\nabla_{\tau} \mathbf{m}\right|^{p} \leq \frac{\mathcal{E}\left(\mathbf{m} ; \mathcal{M}_{t}^{-}\right)}{t}
$$

where $J_{t}$ denotes the Jacobian determinant of $\psi_{t}$. Using the orthogonal decomposition of $\mathbf{R}^{d}$ as $T_{y} \partial \Omega \oplus$ $\mathbf{R n}(y) \simeq T_{y} \partial \Omega \oplus \mathbf{R}$, we compute,

$$
D \psi_{t}(y, s)=\left(\begin{array}{cc}
(1-t s) \operatorname{Id}_{T_{y} \partial \Omega} & -t(y-(y \cdot \mathbf{n}(y)) \mathbf{n}(y)) \\
0 & -t(y \cdot \mathbf{n}(y))
\end{array}\right) .
$$


Hence, $J_{t}(y, s)=t(1-t s)^{d-1}(y \cdot \mathbf{n}(y))=[1+O(t)] t(y \cdot \mathbf{n}(y))$ and the above identity simplifies to

$$
[1+O(t)] \frac{1}{p} \int_{\partial \Omega \times(0,1)}\left|\nabla_{y} \mathbf{v}_{t}\right|^{p}(y, s)(y \cdot \mathbf{n}(y)) d s d \mathcal{H}^{d-1}(y)=\frac{1}{t p} \int_{\mathcal{M}_{t}^{-}}\left|\nabla_{\tau} \mathbf{m}\right|^{p} \leq \frac{\mathcal{E}\left(\mathbf{m} ; \mathcal{M}_{t}^{-}\right)}{t} .
$$

Since $\Omega$ is star-shaped with respect to $B_{\rho}(0)$ the weight $y \cdot \mathbf{n}(y)$ is uniformly bounded from below by a positive constant on $\partial \Omega$. On the other hand, we know from (12) that the right hand side of (16) remains bounded as $t \downarrow 0$. Hence, the family $\left\{\nabla_{y} \mathbf{v}_{t}\right\}_{t \in(0,1 / 2)}$ is bounded in $L^{p}(\partial \Omega \times(0,1))$ and therefore, up to extraction, $\nabla_{y} \mathbf{v}_{t}$ weakly converges in $L^{p}(\partial \Omega \times(0,1))$. We already know from (15) that $\left(\mathbf{v}_{t}\right)_{t}$ converges towards $\mathbf{u}_{0}$ in $\mathcal{D}^{\prime}(\partial \Omega \times(0,1), H)$, and we can thus identify the limit and deduce:

$$
\nabla_{y} \mathbf{v}_{t} \stackrel{t \downarrow 0}{\longrightarrow} \nabla_{y} \mathbf{u}_{0}, \quad \text { weakly in } L^{p}(\partial \Omega \times(0,1)) .
$$

Consequently, $\mathbf{u}_{0} \in W^{1, p}(\partial \Omega, \mathcal{S})$ as claimed. Eventually, by lower semi-continuity of the $L^{p}$-norm under weak convergence, we get the first inequality of (13) by sending $t \downarrow 0$ in (16).

We now establish that the inequalities (13) are in fact identities.

Lemma 24. (Zooming out) The following identities hold.

$$
\frac{1}{p} \int_{\partial \Omega}\left|\nabla \mathbf{u}_{0}(y)\right|^{p}(y \cdot \mathbf{n}(y)) d \mathcal{H}^{d-1}(y)=\lim _{t \downarrow 0} \frac{\mathcal{E}\left(\mathbf{m} ; \mathcal{M}_{t}^{-}\right)}{t}=(d-p) \mathcal{E}(\mathbf{m}) .
$$

Proof. Thanks to the higher regularity of $\mathbf{u}_{0}=\mathbf{m}_{\mid \partial \Omega}$ established in Lemma 23, we are able to use extensions of $\mathbf{m}$ that are only built on $\mathbf{u}_{0}$. Let $s^{\star}>0$ be small enough such that $\psi:(y, s) \in \partial \Omega \times\left(0, s^{\star}\right) \mapsto y+s \mathbf{n}(y) \in \mathbf{R}^{d}$ defines a (bi-Lipschitz) diffeomorphism onto its image $\mathcal{N}$. We set $\mathcal{O}:=\bar{\Omega} \cup \mathcal{N}$ and define the extension $\overline{\mathbf{m}} \in W^{1, p}(\mathcal{O}, \mathcal{S})$ of $\mathbf{m}$ by

$$
\overline{\mathbf{m}}(x)=\left\{\begin{array}{lll}
\mathbf{m}(x) & \text { if } & x \in \Omega, \\
\mathbf{u}_{0}(y) & \text { if } & x=\psi(y, s)
\end{array}\right.
$$

Let $t^{\star}>0$ be such that $\left(1+t^{\star}\right) \Omega \subset \mathcal{O}$, we now define a new inner perturbation of $\mathbf{m}$ by $\mathbf{m}_{t}(x):=\overline{\mathbf{m}}((1+t) x)$ for $x \in \Omega, t \in\left(0, t^{\star}\right)$.

By the local optimality of $\mathbf{m}$, we know that $\mathcal{E}(\mathbf{m}) \leq \mathcal{E}\left(\mathbf{m}_{t}\right)$ for $t>0$ small enough. Then, using the homogeneity of the energy and the splitting of $(1+t) \Omega$ into $\Omega \cup \mathcal{M}_{t}^{+}$, with $\mathcal{M}_{t}^{+}:=(1+t) \Omega \backslash \bar{\Omega}$, we get

$$
\mathcal{E}(\mathbf{m}) \leq \mathcal{E}\left(\mathbf{m}_{t}\right)=(1+t)^{p-d} \mathcal{E}(\mathbf{m} ; \Omega)+(1+t)^{p-d} \mathcal{E}\left(\overline{\mathbf{m}} ; \mathcal{M}_{t}^{+}\right) .
$$

Multiplying by $(1+t)^{d-p}$ leads to

$$
\frac{1}{p} \int_{\mathcal{M}_{t}^{+}}|\nabla \overline{\mathbf{m}}|^{p}(z) d z \geq\left[(1+t)^{d-p}-1\right] \mathcal{E}(\mathbf{m}) .
$$

Dividing by $t$ and letting $t \downarrow 0$, we obtain

$$
\liminf _{t \downarrow 0} \frac{1}{t p} \int_{\mathcal{M}_{t}^{+}}|\nabla \overline{\mathbf{m}}|^{p}(z) d z \geq(d-p) \mathcal{E}(\mathbf{m}) .
$$

But, on $\mathcal{M}_{t}^{+}, \overline{\mathbf{m}}$ is given in terms of $\mathbf{u}_{0}$ which enables us to express the left hand side as a function of $\nabla \mathbf{u}_{0}$. Using the change of variable $z=(1+t s) y$ (as in the previous Lemma), we compute

$$
\frac{1}{t p} \int_{\mathcal{M}_{t}^{+}}|\nabla \overline{\mathbf{m}}|^{p}(z) d z=\frac{1}{p} \int_{0}^{1}\left[\int_{\partial \Omega}|\nabla \overline{\mathbf{m}}|^{p}((1+t s) y)(\mathbf{n}(y) \cdot y) d \mathcal{H}^{d-1}(y)\right](1+t s)^{d-1} d s
$$

Next, from the identity $\overline{\mathbf{m}}(y+s \mathbf{n}(y))=\mathbf{u}_{0}(y)$ and the chain rule, we have for every $\xi \in T_{y} \partial \Omega$,

$$
\xi \cdot \nabla \mathbf{u}_{0}(y)=([\xi+s(\xi \cdot \nabla) \mathbf{n}(y)] \cdot \nabla) \overline{\mathbf{m}}(y+s \mathbf{n}(y)),
$$

and thus

$$
\nabla \overline{\mathbf{m}}(y+s \mathbf{n}(y))=[1+O(s)] \nabla \mathbf{u}_{0}(y) .
$$


Denoting by $\pi$ the orthogonal projection on $\partial \Omega$, this yields

$$
\frac{1}{t p} \int_{\mathcal{M}_{t}^{+}}|\nabla \overline{\mathbf{m}}|^{p}(z) d z=[1+O(t)] \frac{1}{p} \int_{\partial \Omega \times(0,1)}\left|\nabla \mathbf{u}_{0}\right|^{p}(\pi[(1+t s) y])(\mathbf{n}(y) \cdot y) d \mathcal{H}^{d-1}(y) d s .
$$

Now, we let $t \downarrow 0$. Since the family of mappings $(y, s) \mapsto \pi[(1+t s) y]$ converges to $(y, s) \mapsto y$ in $C^{1}(\partial \Omega \times(0,1))$ as $t \downarrow 0$ and since $y \mapsto\left|\nabla \mathbf{u}_{0}\right|^{p}(y)(\mathbf{n}(y) \cdot y)$ belongs to $L^{1}(\partial \Omega)$, we get,

$$
\lim _{t \downarrow 0} \frac{1}{t p} \int_{\check{\Omega}_{t}}|\nabla \overline{\mathbf{m}}|^{p}(z) d z=\frac{1}{p} \int_{\partial \Omega}\left|\nabla \mathbf{u}_{0}\right|^{p}(y)(\mathbf{n}(y) \cdot y) d \mathcal{H}^{d-1}(y) .
$$

Together with (20), this leads to

$$
\frac{1}{p} \int_{\partial \Omega}\left|\nabla \mathbf{u}_{0}\right|^{p}(y)(\mathbf{n}(y) \cdot y) d \mathcal{H}^{d-1}(y) \geq(d-p) \mathcal{E}(\mathbf{m}) .
$$

Using this inequality and (13), we get (18).

The identities of Lemma 24 allow us to convert the weak convergence of Lemma 23 to strong convergence.

Lemma 25. We have the strong convergence

$$
[\nabla \mathbf{m}] \circ \psi_{t} \stackrel{t \downarrow 0}{\longrightarrow} \nabla \mathbf{u}_{0} \quad \text { in } L^{p}(\partial \Omega \times(0,1)),
$$

where we recall the notation $\psi_{t}(y, s):=(1-s t) y$ for $y \in \partial \Omega$ and $s, t \in(0,1)$.

This Lemma plays the role of a regularity result for $\mathbf{m}$ near the boundary. It will be crucial for carrying out the computations in the next sections.

Proof. Using the change of variable $z=\psi_{t}(y, s)=(1-s t) y$ as in the proof of Lemma 23, we get

$$
\frac{1}{p}\left\|[\nabla \mathbf{m}] \circ \psi_{t}\right\|_{L_{\star}^{p}}^{p}=[1+O(t)] \frac{\mathcal{E}\left(\mathbf{m} ; \mathcal{M}_{t}^{-}\right)}{t} .
$$

where we have denoted by $\|\mathbf{v}\|_{L_{\star}^{p}}$ the weighted norm in $L^{p}(\partial \Omega \times(0,1))$ defined by

$$
\|\mathbf{v}\|_{L_{\star}^{p}}:=\left(\int_{\partial \Omega \times(0,1)}|\mathbf{v}|^{p}(y, s)(y \cdot \mathbf{n}(y)) \mathrm{d} \mathcal{H}^{d-1}(y) \mathrm{d} s\right)^{\frac{1}{p}} .
$$

By Lemma 24, we know that

$$
\lim _{t \downarrow 0}\left\|[\nabla \mathbf{m}] \circ \psi_{t}\right\|_{L_{\star}^{p}}=\left\|\nabla \mathbf{u}_{0}\right\|_{L_{\star}^{p}}<\infty .
$$

Thus, there exists $\mathbf{w} \in L^{p}(\partial \Omega \times(0,1))$ and a sequence $t_{k} \downarrow 0$ such that

$$
\left([\nabla \mathbf{m}] \circ \psi_{t_{k}}\right)_{k \in \mathbb{N}} \rightarrow \mathbf{w} \quad \text { weakly in } L^{p}(\partial \Omega \times(0,1)) .
$$

Moreover, by the lower semi-continuity of the $L^{p}$-norm under weak convergence

$$
\|\mathbf{w}\|_{L_{\star}^{p}} \leqslant\left\|\nabla \mathbf{u}_{0}\right\|_{L_{\star}^{p}} .
$$

We now claim that

$$
\mathbf{w}=\nabla \mathbf{u}_{0}
$$

Assuming the claim, we get from (21) and (22)

$$
\left([\nabla \mathbf{m}] \circ \psi_{t_{k}}\right)_{k \in \mathbb{N}} \longrightarrow \nabla \mathbf{u}_{0} \quad \text { strongly in } L^{p}(\partial \Omega \times(0,1)) .
$$

Eventually, since the limit $\nabla \mathbf{u}_{0}$ does not depend on the particular subsequence $\left(t_{k}\right)$, we deduce that the whole family $\left\{[\nabla \mathbf{m}] \circ \psi_{t}\right\}_{t>0}$ converges towards $\nabla \mathbf{u}_{0}$ as $t \downarrow 0$. This establishes the Lemma, assuming (23). 
Let us now establish (23). Using the notation $\partial_{\mathbf{n}} \mathbf{m}\left(\psi_{t}(y, s)\right):=\mathbf{n}(y) \cdot[\nabla \mathbf{m}]\left(\psi_{t}(y, s)\right)$ (notice that this extends the classical normal derivative) and orthogonal decompositions, we have

$$
[\nabla \mathbf{m}] \circ \psi_{t}=\left[\nabla_{\tau} \mathbf{m}\right] \circ \psi_{t}+\mathbf{n} \otimes \partial_{\mathbf{n}} \mathbf{m} \circ \psi_{t}, \quad \mathbf{w}=\mathbf{w}_{\tau}+(\mathbf{w} \cdot \mathbf{n}) \mathbf{n} .
$$

With this decomposition, (21) reads

$$
\left[\nabla_{\tau} \mathbf{m}\right] \circ \psi_{t_{k}} \rightarrow \mathbf{w}_{\tau}, \quad \partial_{\mathbf{n}} \mathbf{m} \circ \psi_{t_{k}} \rightarrow \mathbf{w} \cdot \mathbf{n} \quad \text { both weakly in } L^{p}(\partial \Omega \times(0,1)) \text { as } k \uparrow \infty
$$

Now, in the proof of Lemma 23 we have seen that $\left[\nabla_{\tau} \mathbf{m}\right] \circ \psi_{t}$ weakly converges to $\nabla \mathbf{u}_{0}$ in $L^{p}(\partial \Omega \times(0,1))$, and therefore $\mathbf{w}_{\tau}=\nabla \mathbf{u}_{0}$. Taking into account (23) we end with

$$
\|\mathbf{w}\|_{L_{\star}^{p}} \leqslant\left\|\nabla \mathbf{u}_{0}\right\|_{L_{\star}^{p}}=\left\|\mathbf{w}_{\tau}\right\|_{L_{\star}^{p}} .
$$

Since $\mathbf{w}_{\tau}(y, s)$ and $\mathbf{n}(y)$ are orthogonal in $H$, this yields $\mathbf{w}=\mathbf{w}_{\tau}=\nabla \mathbf{u}_{0}$ and establishes the claim.

Remark 26. The preceding Lemma implies the following strong form of the Neumann boundary conditions:

$$
\left[\partial_{\mathbf{n}} \mathbf{m}\right] \circ \psi_{t} \stackrel{t \downarrow 0}{\longrightarrow} 0 \quad \text { in } L^{p}(\partial \Omega \times(0,1)) .
$$

\subsection{Domain translations (Proof of Theorem 9. iii)}

For the time being, we have considered inner variations produced by dilations of the domain with respect to $x_{0}=0$. When $\Omega$ is the unit ball, these variations do not rule out non-constant mappings of the form $\mathbf{m}(x)=\mathbf{m}_{0}(x /|x|)$. Indeed, for $p<d$ and $\mathbf{m}_{0} \in W^{1, p}\left(\mathbb{S}^{d-1}, \mathcal{S}\right)$ such mappings do belong to $W^{1, p}(\Omega, \mathcal{S})$ and are homogeneous of degree $0,\left(\mathbf{m}_{t}=\mathbf{m}\right)$. Moreover, the identities (18) of Lemma 24 remain true regardless of whether $\mathbf{m}$ is a local minimizer. In such cases our previous computations are not sufficient and looking for second order optimality conditions would not improve the situation if we stick on the same variations. We therefore consider below different inner variations, namely those produced by translations of the domain.

From now on, we assume that the domain $\Omega$ is (still) bounded, convex and of class $C^{2}$. We also assume that $0 \in \Omega$, which implies that $\Omega$ is still star-shaped with respect to some non-empty open ball $B_{\rho}(0)$ and the results of the previous Section 3.1 apply. We also recall that $1<p<d$, and in particular, $d \geq 2$.

We introduce a new extension of $\mathbf{m}$. Let $t^{\star}>0$ be such that

$$
\phi:(y, s) \in \partial \Omega \times\left(-t^{\star}, t^{\star}\right) \longmapsto y+s \mathbf{n}(y) \in \mathbf{R}^{d}
$$

defines a bi-Lipschitz diffeomorphism onto its range. For $t \in\left(0, t^{\star}\right)$, we set,

$$
\mathcal{N}_{t}^{+}:=\phi(\partial \Omega \times(0, t)) \text { and } \mathcal{N}_{t}^{-}:=\phi(\partial \Omega \times(-t, 0)) .
$$

We extend $\mathbf{m}$ on $\left\{x \in \mathbf{R}^{3}: d(x, \Omega)<t^{\star}\right\}=\bar{\Omega} \cup \mathcal{N}_{t^{\star}}^{+}$by setting

$$
\tilde{\mathbf{m}}(x):= \begin{cases}\mathbf{m}(x) & \text { if } x \in \Omega, \\ \mathbf{m}(\phi(y,-s)) & \text { if } x=\phi(y, s),(y, s) \in \partial \Omega \times\left[0, t^{\star}\right) .\end{cases}
$$

Since $\phi$ is a bi-Lipschitz diffeomorphism, we have $\tilde{\mathbf{m}} \in W^{1, p}(\Omega, \mathcal{S})$. Next, for $\theta \in \mathbb{S}^{d-1}$, and $t \in\left(0, t^{\star}\right)$, we set

$$
\mathbf{m}_{t}^{\theta}(x):=\tilde{\mathbf{m}}(x+t \theta) \quad \text { for every } x \in \Omega .
$$

It is clear that $\mathbf{m}_{t}^{\theta} \in W^{1, p}(\Omega, \mathcal{S})$ and by Lemma 21 , there exists $t^{\prime} \in\left(0, t^{\star}\right]$ such that

$$
\mathcal{E}(\mathbf{m}) \leq \mathcal{E}\left(\mathbf{m}_{t}^{\theta}\right) \quad \text { for every } t \in\left(0, t^{\prime}\right) \text { and } \theta \in \mathbb{S}^{d-1} \text {. }
$$

Averaging in $\theta \in \mathbb{S}^{d-1}$, we set for $t \in\left(0, t^{\prime}\right)$,

$$
Q(t):=\frac{1}{t^{2}} \int_{\mathbb{S}^{d-1}}\left[\mathcal{E}\left(\mathbf{m}_{t}^{\theta}\right)-\mathcal{E}(\mathbf{m})\right] d \mathcal{H}^{d-1}(\theta) \geq 0 \quad \text { for } t \in\left(0, t^{\prime}\right) .
$$

The main task of this Section is to establish the following lemma. 
Lemma 27. For $\xi \in T_{y} \partial \Omega$, let us denote by $a_{y}(\xi)=A_{y}(\xi, \xi)$ the quadratic form associated to the second fundamental form $A_{y}$ of $\partial \Omega$ at $y$. We have

$$
\lim _{t \downarrow 0} Q(t)=-\frac{\left|\mathbb{S}^{d-1}\right|}{2 d} \int_{\partial \Omega} a_{y}\left(\nabla \mathbf{u}_{0}(y)\right)\left|\nabla \mathbf{u}_{0}\right|^{p-2}(y) d \mathcal{H}^{d-1}(y) .
$$

The factor $a_{y}\left(\nabla \mathbf{u}_{0}(y)\right)$ in the integrand of (27) deserves some comment. Since $\Omega$ is of class $C^{2}, a_{y}$ is well defined as a quadratic form on $T_{y} \partial \Omega$. As usual, we extend this quadratic form to $\mathbf{R}^{d}$ by setting $a_{y}(\xi):=a_{y}\left(\pi_{y} \xi\right)$ where $\pi_{y}$ denotes the orthogonal projection onto $T_{y} \partial \Omega$. Next, given, any Hilbertian basis $\mathcal{B}$ of $H$, we can extend the domain $a_{y}$ to continuous linear forms $V: \mathbf{R}^{d} \rightarrow H$. Indeed, writing the decomposition of $V$ into the Hilbertian basis as $V(z)=\sum_{b \in \mathcal{B}} V_{b}(z) b$, for $z \in \mathbf{R}^{d}$, we set

$$
a_{y}(V):=\sum_{b \in \mathcal{B}} a_{y}\left(V_{b}\right) .
$$

With this convention, the integrand in the right hand side of (27) is well defined with

$$
a_{y}\left(\nabla \mathbf{u}_{0}(y)\right)=\sum_{b \in \mathcal{B}} a_{y}\left(\nabla\left(\mathbf{u}_{0}(y) \cdot b\right)\right)=\sum_{b \in \mathcal{B}} A_{y}\left(\nabla\left(\mathbf{u}_{0}(y) \cdot b\right), \nabla\left(\mathbf{u}_{0}(y) \cdot b\right)\right) .
$$

We postpone the proof of Lemma 27, and show first that it implies Theorem 9.iii.

Proof. (of Theorem 9, iii.) We assume that $\Omega$ is uniformly convex or, at least, that $a_{y}$ is coercive for almost every $y \in \partial \Omega$ (see Remark 11). Assuming that (27) holds, with (26), this leads to

$$
\int_{\partial \Omega} a_{y}\left(\nabla \mathbf{u}_{0}\right)\left|\nabla \mathbf{u}_{0}\right|^{p-2} d \mathcal{H}^{d-1}(y) \leq 0 .
$$

Since $a_{y}$ is coercive almost everywhere on $\partial \Omega$, we see that $\nabla \mathbf{u}_{0}$ vanishes in $L^{p}(\partial \Omega)$. The identities of Lemma 24 then lead to $\mathcal{E}(\mathbf{m})=0$ and $\mathbf{m}$ is constant, as claimed.

Proof. (of Lemma 27) To lighten notation, we set

By Fubini, we rewrite $Q(t)$ as

$$
q(z):=\frac{1}{p}|\nabla \tilde{\mathbf{m}}|^{p}(z) \quad \text { for } z \in \bar{\Omega} \cup \mathcal{N}_{t^{\star}}^{+} .
$$

with

$$
Q(t)=\frac{1}{t^{2}} \int_{\mathbf{R}^{d}} w_{t}(z) q(z) d z
$$

$$
w_{t}(z)=\mathcal{H}^{d-1}\left(\left\{\theta \in \mathbb{S}^{d-1}: z \in \Omega+t \theta\right\}\right)-\mathcal{H}^{d-1}\left(\mathbb{S}^{d-1}\right) \mathbf{1}_{\Omega}(z) .
$$

Obviously, if $z \in \Omega$ is such that $d(z, \partial \Omega) \geq t$, then $w_{t}(z)=0$. For $z \in \partial \Omega+B_{t}(0)$, we distinguish the cases $z \in \mathcal{N}_{t}^{+}$and $z \in \mathcal{N}_{t}^{-}$.

$$
w_{t}(z)=\left\{\begin{array}{l}
\mathcal{H}^{d-1}\left(\left\{\theta \in \mathbb{S}^{d-1}: z \in \Omega+t \theta\right\}\right) \quad \text { if } z \in \mathcal{N}_{t}^{+}, \\
-\mathcal{H}^{d-1}\left(\left\{\theta \in \mathbb{S}^{d-1}: z \in\left[\mathbf{R}^{d} \backslash \Omega\right]+t \theta\right\}\right) \quad \text { if } z \in \mathcal{N}_{t}^{-}, \\
0 \quad \text { if } z \in \mathbf{R}^{d} \backslash\left[\mathcal{N}_{t}^{+} \cup \mathcal{N}_{t}^{-}\right] .
\end{array}\right.
$$

Using the change of variables $z=\phi(y, r t)=y+r t \mathbf{n}(y)$, we obtain

$$
\left.Q(t)=\frac{1}{t} \int_{\partial \Omega} \int_{-1}^{1} w_{t} \circ \phi(y, r t) q \circ \phi(y, r t)\right) J_{\phi}(y, r t) d r d \mathcal{H}^{d-1}(y),
$$

where $J_{\phi}$ denotes the Jacobian determinant of $\phi$. We rewrite this expression under the form

$$
Q(t)=\frac{1}{t} \int_{\partial \Omega} \int_{0}^{1}\left\{\left[\left(w_{t} \circ \phi\right)(q \circ \phi) J_{\phi}\right](y, r t)-\left[\left(w_{t} \circ \phi\right)(q \circ \phi) J_{\phi}\right](y,-r t)\right\} d r d \mathcal{H}^{d-1}(y) .
$$


In order to obtain the limit of this integral as $t \downarrow 0$, we compute the first order expansions in $t$ of $w_{t}, q$ and $J_{\phi}$.

- First, using the orthogonal decomposition $\mathbf{R}^{d}=T_{y} \partial \Omega \oplus \mathbf{R} \mathbf{n}(y) \simeq T_{y} \partial \Omega \oplus \mathbf{R}$, we compute

$$
D \phi(y, s)=\left(\begin{array}{cc}
\operatorname{Id}_{T_{y} \partial \Omega}+s D \mathbf{n}(y) & 0 \\
0 & 1
\end{array}\right)
$$

which gives, uniformly in $y \in \partial \Omega$ and $r \in(-1,1)$,

$$
J_{\phi}(y, r t)=1+r t \kappa(y)+o(t),
$$

where $\kappa(y)=\operatorname{Tr} A_{y}$ denotes the total curvature of $\partial \Omega$ at $y$.

- Next, for $r \in(0,1)$, using $\tilde{\mathbf{m}}(\phi(y, r t))=\mathbf{m}(\phi(y,-r t))$, we compute

$$
\begin{aligned}
D \tilde{\mathbf{m}}(\phi(y, r t)) & =D \mathbf{m}(\phi(y,-r t)) \cdot D \phi(y,-r t) \cdot D \phi^{-1}(y, r t) \\
& =D \mathbf{m}(\phi(y,-r t)) \cdot[\operatorname{Id}-2 r t D \mathbf{n}(y)+o(t)] .
\end{aligned}
$$

This yields the expansion, using the shorter notation $y^{ \pm}=y \pm r t \mathbf{n}(y)$

$$
q\left(y^{+}\right)=q\left(y^{-}\right)-2 r t\left(D \mathbf{m}^{T}\left(y^{-}\right) \cdot D \mathbf{n}(y) \cdot D \mathbf{m}\left(y^{-}\right)\right)|\nabla \tilde{\mathbf{m}}|^{p-2}\left(y^{-}\right)+o(t) q\left(y^{-}\right)
$$

that we rewrite as

$$
q\left(y^{+}\right)=q\left(y^{-}\right)-2 r t a_{y}\left(\nabla \mathbf{m}\left(y^{-}\right)\right)|\nabla \mathbf{m}|^{p-2}\left(y^{-}\right)+o(t) q\left(y^{-}\right),
$$

uniformly in $y \in \partial \Omega, r \in(0,1)$.

- Eventually, we establish that

$$
\frac{w_{t}(\phi(y, \pm r t))}{\left|\mathbb{S}^{d-2}\right|}= \pm \Theta_{0}(r)-\Theta_{1}(r) \kappa(y) t+o(t),
$$

holds uniformly in $y \in \partial \Omega, r \in(0,1)$ and with the notation

$$
\Theta_{0}(r):=\int_{0}^{\operatorname{acos} r}(\sin \varphi)^{d-2} d \varphi, \quad \Theta_{1}(r):=\frac{1}{2(d-1)}\left(1-r^{2}\right)^{\frac{d-1}{2}} .
$$

Proof. (of (32)) Let $y \in \partial \Omega$. Without loss of generality, we use local coordinates for which $y=0, \mathbf{n}(y)=\mathbf{e}_{d}$ and we identify $T_{y} \partial \Omega$ with $\mathbf{R}^{d-1}$. By definition, for $t$ small enough,

$$
\begin{array}{r}
w_{t}(\phi(y,+r t))=\mathcal{H}^{d-1}\left(\left\{\theta \in \mathbb{S}^{d-1}: r t \mathbf{e}_{d}+t \theta \in \Omega\right\}\right), \\
w_{t}(\phi(y,-r t))=-\mathcal{H}^{d-1}\left(\left\{\theta \in \mathbb{S}^{d-1}:-r t \mathbf{e}_{d}+t \theta \in \mathbf{R}^{d} \backslash \bar{\Omega}\right\}\right) .
\end{array}
$$

Since $\Omega$ is of class $C^{2}$, using a local chart, we can parameterize locally $\partial \Omega$ as the graph of a $C^{2}$ concave function. Calling $D_{\rho}$ the $(d-1)$-ball $D_{\rho}=T_{y} \partial \Omega \cap B_{\rho}(y) \subset \mathbf{R}^{d-1}$, there exists $\rho>0$ and $h_{y} \in C^{2}\left(D_{\rho}, \mathbf{R}\right)$ such that

$$
\partial \Omega \cap\left[D_{\rho} \times(-\rho, \rho)\right]=\left\{\left(\xi, h_{y}(\xi)\right) ; \xi \in D_{\rho}\right\} .
$$

With our hypotheses, $h_{y}$ satisfies

$$
h_{y}(0)=0, \quad \nabla h_{y}(0)=0, \quad \text { and } \quad D^{2} h_{y}(0)(\xi, \eta)=-A_{y}(\xi, \eta) \quad \text { for every } \xi, \eta \in \mathbf{R}^{d-1}=T_{y} \partial \Omega .
$$

Moreover ( $\partial \Omega$ being compact) there exists $C_{\Omega} \geq 0$ that only depends on $\Omega$ such that uniformly in $y \in \partial \Omega$

$$
\left\|D^{2} h_{y}\right\|_{\infty} \leq C_{\Omega} .
$$

Notice also that $\rho$ only depends on $\Omega$.

We now estimate (33). Let $\xi \in \mathbb{S}^{d-2} \subset \mathbf{R}^{d-1}$ and let us study the intersection of $\left\{\theta \in \mathbb{S}^{d-1} ;-r t \mathbf{e}_{d}+t \theta \in\right.$ $\left.\mathbf{R}^{d} \backslash \bar{\Omega}\right\}$ with the half-plane $\mathbf{R} \mathbf{e}_{d} \oplus \mathbf{R}_{+} \xi$. For this, we consider the following parametrization of the semicircle $\mathbb{S}^{d-1} \cap\left[\mathbf{R} \mathbf{e}_{d} \oplus \mathbf{R}_{+} \xi\right]$,

$$
\mathbf{e}_{\varphi}:=(\cos \varphi) \mathbf{e}_{d}+(\sin \varphi) \xi, \quad \text { for } \varphi \in[0, \pi]
$$




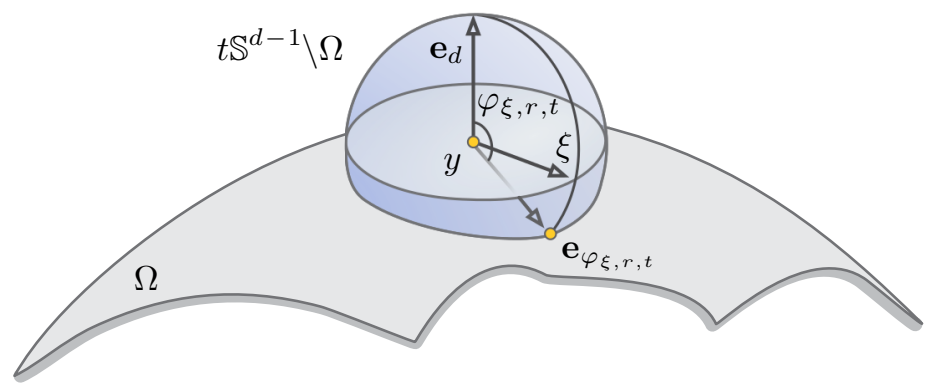

Figure 1.

For $r \in(0,1),-r t \mathbf{e}_{d}+t \mathbf{e}_{\varphi} \in \mathbf{R}^{d} \backslash \bar{\Omega}$ if and only if

$$
f_{\xi, r, t}(\varphi):=-t \cos \varphi+r t+h_{y}(t(\sin \varphi) \xi)<0 .
$$

Performing the Taylor expansion of $f_{\xi, r, t}(\varphi)$ at $t=0$ leads to

$$
\frac{f_{\xi, r, t}(\varphi)}{t}=-\cos \varphi+r+\frac{t \sin ^{2} \varphi}{2} D^{2} h_{y}(0)(\xi, \xi)+o(t) .
$$

Now, noticing that $f_{\xi, r, t}(0) / t=(r-1)<0$ and $f_{\xi, r, t}(\pi) / t=(1-r)>0$, we see that $f_{\xi, r, t}$ changes sign only once for $t$ sufficiently small. Consequently, $f_{\xi, r, t}(\varphi)<0$ on $\left[0, \varphi_{\xi, r, t}\right)$ where $\varphi_{\xi, r, t} \in(0, \pi)$ is the unique solution in $(0, \pi)$ of $f_{\xi, r, t}(\varphi)=0$ (see Figure 2). The Taylor expansion of $\varphi_{\xi, r, t}$ writes

$$
\varphi_{\xi, r, t}=\operatorname{acos}(r)-\frac{t}{2} D^{2} h_{y}(0)(\xi, \xi)(\sin \operatorname{acos}(r))+o(t) .
$$

Therefore, integrating in $\xi \in \mathbb{S}^{d-2}$, we get

$$
\begin{aligned}
w_{t}(\phi(y,-r t))= & -\int_{\mathbb{S}^{d-2}}\left\{\int_{0}^{\varphi_{\xi, r, t}}(\sin \varphi)^{d-2} d \varphi\right\} d \mathcal{H}^{d-2}(\xi) \\
= & -\mathcal{H}^{d-2}\left(\mathbb{S}^{d-2}\right) \int_{0}^{\operatorname{acos} r}(\sin \varphi)^{d-2} d \varphi \\
& +\frac{t}{2}\left(\int_{\mathbb{S}^{d-2}} D^{2} h_{y}(0)(\xi, \xi) d \mathcal{H}^{d-2}(\xi)\right)\left(1-r^{2}\right)^{\frac{d-1}{2}}+o(t) .
\end{aligned}
$$

By the use of the identity $\int_{\mathbb{S}^{d-2}}(\mathbf{e} \cdot \xi)^{2} d \mathcal{H}^{d-2}(\xi)=\left|\mathbb{S}^{d-2}\right| /(d-1)$ for all $\mathbf{e} \in \mathbb{S}^{d-2}$ and the diagonalization of $D^{2} h_{y}(0)$ in an orthonormal basis, we have

$$
\int_{\mathbb{S}^{d-2}} D^{2} h_{y}(0)(\xi, \xi) d \mathcal{H}^{d-2}(\xi)=\frac{\mathcal{H}^{d-2}\left(\mathbb{S}^{d-2}\right)}{d-1} \operatorname{Tr} D^{2} h_{y}(0)=-\frac{\mathcal{H}^{d-2}\left(\mathbb{S}^{d-2}\right)}{d-1} \kappa(y) .
$$

Substituting this identity in the expression above, we obtain the expected expansion of $w_{t}(\phi(y,-r t))$. The computation for $w_{t}(\phi(y, r t))$ is similar and can be obtained by substituting $-h_{y}$ for $h_{y}$. This establishes (32).

We are now able to compute the limit of $Q(t)$ as $t \downarrow 0$. Plugging the expansions (30), (31) and (32) in (29), we get:

$$
\begin{array}{r}
Q(t)=-\left|\mathbb{S}^{d-2}\right|\left\{\int_{\partial \Omega} \kappa(y) \int_{0}^{1}\left(\Theta_{1}(r)-r \Theta_{0}(r)\right) q(\phi(y,-r t)) d r d \mathcal{H}^{d-1}(y)\right. \\
+\int_{\partial \Omega} \int_{0}^{1} 2 r \Theta_{0}(r) a_{y}(\nabla \mathbf{m}(\phi(y,-r t)))|\nabla \mathbf{m}|^{p-2}(y) d r d \mathcal{H}^{d-1}(y) \\
\left.+o(1) \int_{\partial \Omega} \int_{0}^{1} q(\phi(y,-r t)) d r d \mathcal{H}^{d-1}(y)\right\} .
\end{array}
$$


To justify the passage to the limit $t \downarrow 0$, we prove the following property which is a direct consequence of Lemma 25 and of the convexity of the domain.

Lemma 28. Let $\left\{\Psi_{t}\right\}_{0 \leq t}$ be the family of functions defined on $\partial \Omega \times(0,1)$ by

$$
\Psi_{t}(y, r)=y-r t \mathbf{n}(y)=\phi(y,-r t) .
$$

Then $[\nabla \mathbf{m}] \circ \Psi_{t} \stackrel{t \downarrow 0}{\longrightarrow} \nabla \mathbf{u}_{0}$ in $L^{p}(\partial \Omega \times(0,1))$.

Proof. Let $\lambda:=1 / \min \{|y| ; y \in \partial \Omega\}>0$. We use the change of variable $(y, r)=\Psi_{t}^{-1} \circ \psi_{\lambda t}(y, s)$, where we recall that $\psi_{\lambda t}(y, s)=(1-s \lambda t) y$ for $y \in \partial \Omega, s, t \in(0,1)$. We have

$$
\begin{aligned}
\int_{\partial \Omega \times(0,1)} \mid[\nabla \mathbf{m}] \circ & \Psi_{t}(y, r)-\left.\nabla \mathbf{u}_{0}(y)\right|^{p} d \mathcal{H}^{d-1}(y) d r \\
& =\int_{\psi_{\lambda t}^{-1}\left(\tilde{\Omega}_{t}\right)}\left|[\nabla \mathbf{m}] \circ \psi_{\lambda t}(y, s)-\nabla \mathbf{u}_{0}(y)\right|^{p} J_{t}(y, s) d \mathcal{H}^{d-1}(y) d s .
\end{aligned}
$$

where $J_{t}$ denotes the Jacobian determinant of $\Psi_{t}^{-1} \circ \psi_{\lambda t}$. We easily check by direct computation that $J_{t}$ is uniformly bounded. By Lemma 25, the right hand side integral goes to 0 as $t \downarrow 0$.

Passing to the limit $t \downarrow 0$ in (34) we get (using Lemma 28 in the three integrals)

$$
\begin{aligned}
\lim _{t \downarrow 0} Q(t)=-\left|\mathbb{S}^{d-2}\right| & \left\{\left(\int_{0}^{1} \Theta_{1}(r)-r \Theta_{0}(r) d r\right) \int_{\partial \Omega} H(y) \frac{\left|\nabla \mathbf{u}_{0}\right|^{p}(y)}{p} d \mathcal{H}^{d-1}(y)\right. \\
+ & \left.\left(\int_{0}^{1} 2 r \Theta_{0}(r) d r\right) \int_{\partial \Omega} a_{y}\left(\nabla \mathbf{u}_{0}(y)\right)\left|\nabla \mathbf{u}_{0}\right|^{p-2}(y) d \mathcal{H}^{d-1}(y)\right\} .
\end{aligned}
$$

Let us now compute the integrals in $r$. Using Fubini and then integrating by parts, we get:

$$
\begin{aligned}
\int_{0}^{1} r \Theta_{0}(r) d r & =\int_{0}^{1} \int_{0}^{\operatorname{acos} r}(\sin \varphi)^{d-2} d \varphi d r \\
& =\int_{0}^{\pi / 2}(\sin \varphi)^{d-2}\left(\int_{0}^{\cos \varphi} r d r\right) d \varphi \\
& =\frac{1}{2(d-1)} \int_{0}^{\pi / 2}(\sin \varphi)^{d} d \varphi
\end{aligned}
$$

while, using the change of variable $r=\cos \varphi$,

$$
\int_{0}^{1} \Theta_{1}(r) d r=\frac{1}{2(d-1)} \int_{0}^{\pi / 2}(\sin \varphi)^{d} d \varphi=\int_{0}^{1} r \Theta_{0}(r) d r .
$$

Calling $W_{d}=\int_{0}^{\pi / 2}(\sin \varphi)^{d} d \varphi$ the Wallis integrals we get

$$
\lim _{t \downarrow 0} Q(t)=-\frac{\left|\mathbb{S}^{d-2}\right| W_{d}}{d-1} \int_{\partial \Omega} a_{y}\left(\nabla \mathbf{u}_{0}(y)\right)\left|\nabla \mathbf{u}_{0}\right|^{p-2}(y) d \mathcal{H}^{d-1}(y) .
$$

This expression can be further simplified by using the classical relation $W_{d} /(d-1)=W_{d-2} / d$ and the identity $\left|\mathbb{S}^{d-1}\right|=2\left|\mathbb{S}^{d-2}\right| W_{d-2}$. We thus have $\left|\mathbb{S}^{d-2}\right| W_{d} /(d-1)=\left|\mathbb{S}^{d-1}\right| /(2 d)$, leading to (27). This completes the proof of Lemma 27 and thus of Theorem 9.

\section{Proof of Theorem 14}

Let $\Omega \subset \mathbf{R}^{d}, d \geq 3$, let $\mathcal{S}$ be a closed subset of some Hilbert space $H$ and $\mathcal{F}: L^{2}(\Omega, H) \rightarrow \mathbf{R}$. The proof of Theorem 14 proceeds as follows. In Section 4.1, considering $\mathcal{D}_{\varepsilon}$ as a perturbation of $\mathcal{E}$ (in the case $p=2$ ), we go through the same steps as for the unperturbed case to obtain an inequality of the form

$$
\mathcal{D}(\mathbf{m}) \leq \varepsilon^{2}\left[L(\mathbf{m})+L^{\prime}(\mathbf{m})+Q(\mathbf{m})\right]
$$


where, roughly speaking, $L(\mathbf{m}), L^{\prime}(\mathbf{m})$ and $Q^{\prime}(\mathbf{m})$ are respectively linear in $\mathbf{k}[\mathbf{m}] \otimes \nabla \mathbf{m}, \quad \nabla[\mathbf{k}[\mathbf{m}]] \otimes \nabla \mathbf{m}$ and $D \mathbf{k}[\mathbf{m}] \otimes \nabla \mathbf{m}$. The estimates of hypothesis (H2) then lead to

$$
\left|L(\mathbf{m})+L^{\prime}(\mathbf{m})\right| \leq \kappa_{L}\|\nabla \mathbf{m}\|_{L^{2}}\left(1+\|\nabla \mathbf{m}\|_{L^{2}}\right), \quad|Q(\mathbf{m})| \leq \kappa_{Q}\|\nabla \mathbf{m}\|_{L^{2}}^{2} .
$$

Simplifying, we obtain $\|\nabla \mathbf{m}\|_{L^{2} \leq} \frac{2 \kappa_{L}}{1-2\left(\kappa_{Q}+\kappa_{L}\right) \varepsilon^{2}} \varepsilon^{2}$ which proves Theorem 14.i.

Eventually, in Section 4.2, we assume that (H3-H4) hold and we compare $\mathcal{D}_{\varepsilon}(\mathbf{m})$ with $\mathcal{D}_{\varepsilon}\left(R_{t} \mathbf{m}\right)$ when $\left\{R_{t}\right\}$ is a continuous group of isometries of $\mathcal{S}$. Since the Dirichlet energy is invariant by isometry of the target we deduce that $\langle\mathbf{m}\rangle$ is almost a critical point of $\mathcal{F}$. This fact and hypothesis (H3) then lead to the quadratic estimate $\left|L(\mathbf{m})+L^{\prime}(\mathbf{m})\right| \leq \kappa_{Q}^{\prime}\|\nabla \mathbf{m}\|_{L^{2}}^{2}$ instead of the linear one we had before. As a consequence

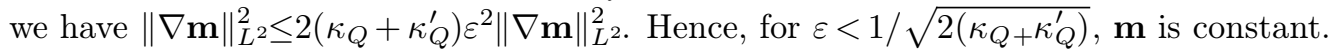

\subsection{Proof of Theorem 14.i. (inner variations)}

In this subsection, we assume that Hypotheses (H1-H2) hold. Let us first state the counterpart of Lemma 23.

Lemma 29. The trace $\mathbf{u}_{0}$ of $\mathbf{m}$ on $\partial \Omega$ belongs to $H^{1}(\partial \Omega, \mathcal{S})$, with the estimate

$$
\frac{1}{2} \int_{\partial \Omega}\left|\nabla u_{0}(y)\right|^{p}(y \cdot \mathbf{n}(y)) d \mathcal{H}^{2}(y)+\varepsilon^{2} \int_{\Omega} \mathbf{k}[\mathbf{m}](x) \cdot(x \cdot \nabla \mathbf{m}(x)) d x \leq \mathcal{D}(\mathbf{m}) .
$$

Proof. We proceed as in the proofs of Lemmas 15 and 23, use the same notation and skip the details. By local optimality of $\mathbf{m}, \mathcal{D}_{\varepsilon}(\mathbf{m}) \leq \mathcal{D}_{\varepsilon}\left(\mathbf{m}_{t}\right)$ for $t>0$ small enough, where $\mathbf{m}_{t}(x)=\mathbf{m}((1-t) x)$ for $t \in[0,1)$ and $x \in \Omega$. Since

$$
\mathcal{D}_{\varepsilon}\left(\mathbf{m}_{t}\right)=(1-t)^{-1} \mathcal{D}(\mathbf{m} ;(1-t) \Omega)+\varepsilon^{2} \mathcal{F}\left(\mathbf{m}_{t}\right),
$$

proceeding as in Lemma 15, we are led to

$$
\frac{\mathcal{D}\left(\mathbf{m} ; \mathcal{M}_{t}^{-}\right)}{t} \leq \mathcal{D}(\mathbf{m})+\varepsilon^{2}(1-t) \frac{\mathcal{F}\left(\mathbf{m}_{t}\right)-\mathcal{F}(\mathbf{m})}{t},
$$

(with $\mathcal{M}_{t}^{-}=\Omega \backslash(1-t) \bar{\Omega}$ ). Using the differentiability of $\mathcal{F}$, we rewrite the last term as

$$
\frac{\mathcal{F}\left(\mathbf{m}_{t}\right)-\mathcal{F}(\mathbf{m})}{t}=\left(\mathbf{k}[\mathbf{m}], \frac{\mathbf{m}_{t}-\mathbf{m}}{t}\right)_{L^{2}}+o\left(\left\|\frac{\mathbf{m}_{t}-\mathbf{m}}{t}\right\|_{L^{2}}\right) .
$$

Now, since $\mathbf{m} \in H^{1}(\Omega, H)$, we have for almost every $x \in \Omega$,

$$
\frac{\mathbf{m}_{t}-\mathbf{m}}{t}(x)=-\int_{0}^{1} x \cdot \nabla \mathbf{m}((1-t s) x) d s=-x \cdot \nabla \mathbf{m}(x)-\int_{0}^{1} x \cdot[\nabla \mathbf{m}((1-t s) x)-\nabla \mathbf{m}(x)] d s .
$$

Squaring, integrating on $\Omega$ and then using Jensen inequality and Fubini, we get

$$
\begin{aligned}
\int_{\Omega}\left|\frac{\mathbf{m}_{t}(x)-\mathbf{m}(x)}{t}+x \cdot \nabla \mathbf{m}(x)\right|^{2} & \leq \int_{\Omega}|x|^{2} \int_{0}^{1}|\nabla \mathbf{m}((1-\mathrm{ts}) x)-\nabla \mathbf{m}(x)|^{2} d s d x \\
& \leq \int_{0}^{1}\left(\int_{\Omega}|\nabla \mathbf{m}((1-\mathrm{ts}) x)-\nabla \mathbf{m}(x)|^{2} d x\right) d s .
\end{aligned}
$$

Since $\nabla \mathbf{m} \in L^{2}\left(\Omega, H^{d}\right)$, the family of maps $\left\{\mathbf{v}_{\lambda}\right\}_{1 / 2<\lambda<1}$ defined by $\mathbf{v}_{\lambda}(x):=\nabla \mathbf{m}(\lambda x)$ is relatively compact in $L^{2}(\Omega)$ (proceed as in the proof of Lemma 21). We already know that $\mathbf{v}_{\lambda} \stackrel{\lambda \uparrow 1}{\longrightarrow} \nabla \mathbf{m}$ in the sense of distributions, so this convergence also holds in $L^{2}(\Omega)$. In particular, the last integral tends to 0 as $t \downarrow 0$ and we conclude that $(1 / t)\left[\mathbf{m}_{t}-\mathbf{m}\right] \stackrel{t \downarrow 0}{\longrightarrow}(x \mapsto-x \cdot \nabla \mathbf{m}(x))$ in $L^{2}(\Omega)$.

Coming back to (37), we get

$$
\frac{\mathcal{F}\left(\mathbf{m}_{t}\right)-\mathcal{F}(\mathbf{m})}{t}=-\int_{\Omega} \mathbf{k}[\mathbf{m}](x) \cdot(x \cdot \nabla \mathbf{m}(x)) d x+o(1) .
$$


In particular, the right hand side of (36) is bounded as $t \downarrow 0$ and we have,

$$
\varlimsup_{t \downarrow 0} \frac{\mathcal{D}\left(\mathbf{m} ; \mathcal{M}_{t}^{-}\right)}{t} \leq \mathcal{D}(\mathbf{m})-\varepsilon^{2} \int_{\Omega} \mathbf{k}[\mathbf{m}](x) \cdot(x \cdot \nabla \mathbf{m}(x)) d x .
$$

We then conclude as in the proof of Lemma 23.

Next, we revisit Lemma 24.

Lemma 30. The following identities hold.

$$
\frac{1}{2} \int_{\partial \Omega}\left|\nabla \mathbf{u}_{0}(y)\right|^{2}(y \cdot \mathbf{n}(y)) d \mathcal{H}^{2}(y)+\varepsilon^{2} \int_{\Omega} \mathbf{k}[\mathbf{m}](x) \cdot(x \cdot \nabla \mathbf{m}(x)) d x=\lim _{t \downarrow 0} \frac{\mathcal{D}\left(\mathbf{m} ; \mathcal{M}_{t}^{-}\right)}{t}=\mathcal{D}(\mathbf{m}) .
$$

Moreover $\mathbf{m}$ satisfies the conclusions of Lemmas 25 and 28 with $p=2$.

Proof. We proceed as in the proof of Lemma 24, we extend $\mathbf{m}$ on a neighborhood $\mathcal{O} \supset \bar{\Omega}$ by the function $\overline{\mathbf{m}}$ defined by $(19)$. We then set $\mathbf{m}_{t}(x)=\overline{\mathbf{m}}((1+t) x)$ for $x \in \Omega$ and $t \in\left(0, t^{\star}\right)$. The optimality of $\mathbf{m}$ now leads to

$$
\mathcal{D}(\mathbf{m}) \leq \frac{\mathcal{D}(\overline{\mathbf{m}} ;(1+t) \Omega \backslash \bar{\Omega})}{t}+\varepsilon^{2}(1+t) \frac{\mathcal{F}\left(\mathbf{m}_{t}\right)-\mathcal{F}(\mathbf{m})}{t}
$$

Since $\overline{\mathbf{m}} \in H^{1}(\Omega, \mathcal{O})$, we obtain, as in the previous proof,

$$
\frac{\mathcal{F}\left(\mathbf{m}_{t}\right)-\mathcal{F}(\mathbf{m})}{t}=\int_{\Omega} \mathbf{k}[\mathbf{m}](x) \cdot(x \cdot \nabla \mathbf{m}(x)) d x+o(1) .
$$

The proof of (38) is now a copy of that of Lemma 24. Lemmas 25 and 28 follow since their proofs only rely on the last equality of $(38)$ and on the convexity of $\Omega$.

Eventually we consider translations of the domain as in Section 3.2.

Lemma 31. We have

$$
\int_{\partial \Omega} a_{y}\left(\nabla \mathbf{u}_{0}(y)\right) d \mathcal{H}^{2}(y) \leq \varepsilon^{2} \int_{\Omega} \sum_{i=1}^{d}\left(\left\{D \mathbf{k}[\mathbf{m}] \cdot \partial_{i} \mathbf{m}\right\} \cdot \partial_{i} \mathbf{m}-\partial_{i}\{\mathbf{k}[\mathbf{m}]\} \cdot \partial_{i} \mathbf{m}\right) .
$$

Proof. We use the notation of Section 3.2, define

$$
Q(t):=\frac{1}{t^{2}} \int_{\mathbb{S}^{d-1}}\left[\mathcal{D}\left(\mathbf{m}_{t}^{\theta}\right)-\mathcal{D}(\mathbf{m})\right] d \mathcal{H}^{d-1}(\theta), \quad R(t):=\frac{1}{t^{2}} \int_{\mathbb{S}^{d-1}}\left[\mathcal{F}\left(\mathbf{m}_{t}^{\theta}\right)-\mathcal{F}(\mathbf{m})\right] d \mathcal{H}^{d-1}(\theta)
$$

and set $Q_{\varepsilon}:=Q+\varepsilon^{2} R$.

By local optimality of $\mathbf{m}$, we know that for $t>0$ small enough $Q_{\varepsilon}(t) \geq 0$. The computations of Section 3.2 leading to (27) remain valid in the present context. In particular, by Lemma 30 we can use Lemma 28. Consequently,

$$
\lim _{t \downarrow 0} Q(t)=-\frac{\left|\mathbb{S}^{d-1}\right|}{2} \int_{\partial \Omega} a_{y}\left(\nabla \mathbf{u}_{0}(y)\right) d \mathcal{H}^{d-1}(y) .
$$

Let us now compute the limit of $R(t)$ as $t$ tends to 0 . Since $\mathcal{F}$ is continuously differentiable, we have for $t>0$ small enough and every $\theta \in \mathbb{S}^{d-1}$,

$$
\frac{\mathcal{F}\left(\mathbf{m}_{t}^{\theta}\right)-\mathcal{F}(\mathbf{m})}{t}=\int_{0}^{1} \int_{\Omega} \mathbf{k}\left[\mathbf{m}_{s t}^{\theta}\right](x) \cdot\left(\theta \cdot \nabla \mathbf{m}_{s t}^{\theta}(x)\right) d x d s .
$$

Rewriting the integrand as

$$
\mathbf{k}\left[\mathbf{m}_{s t}^{\theta}\right] \cdot\left(\theta \cdot \nabla \mathbf{m}_{s t}^{\theta}\right)=\mathbf{k}\left[\mathbf{m}_{s t}^{\theta}\right] \cdot(\theta \cdot \nabla \mathbf{m})+\mathbf{k}\left[\mathbf{m}_{s t}^{\theta}\right] \cdot\left(\theta \cdot \nabla\left\{\mathbf{m}_{s t}^{\theta}-\mathbf{m}\right\}\right),
$$


and integrating in $\theta \in \mathbb{S}^{d-1}$, we obtain,

$$
\begin{array}{r}
R(t)=\frac{1}{2 t} \int_{\mathbb{S}^{d-1}} \int_{0}^{1} \int_{\Omega}\left\{\mathbf{k}\left[\mathbf{m}_{s t}^{\theta}\right]-\mathbf{k}\left[\mathbf{m}_{s t}^{-\theta}\right]\right\}(x) \cdot(\theta \cdot \nabla \mathbf{m})(x) d x d s d \mathcal{H}^{d-1}(\theta) \\
+\frac{1}{t} \int_{\mathbb{S}^{d-1}} \int_{0}^{1} \int_{\Omega} \mathbf{k}\left[\mathbf{m}_{s t}^{\theta}\right](x) \cdot\left(\theta \cdot \nabla\left\{\mathbf{m}_{s t}^{\theta}-\mathbf{m}\right\}\right)(x) d x d s d \mathcal{H}^{d-1}(\theta) \\
=: R_{1}(t)+R_{2}(t) .
\end{array}
$$

For the first term, we notice that $\tilde{\mathbf{m}} \in H^{1}\left(\left\{\Omega+B_{t^{*}}(0)\right\}\right)$ implies that $\xi \in \mathbf{R}^{d} \mapsto \tilde{\mathbf{m}}(\xi+\cdot) \in L^{2}(\Omega$, $\left.\mathbf{R}^{d}\right)$ is differentiable in $B_{t^{\star}}(0)$ with differential $\nabla \tilde{\mathbf{m}}(\xi+\cdot)$. Since $\nabla \tilde{\mathbf{m}} \in L^{2}\left(\Omega+B_{t^{\star}}(0)\right)$, we deduce that $\{(\tilde{\mathbf{m}}(\xi+\cdot)-\mathbf{m}) /|\xi|\}_{\xi \in B_{t^{\star}}(0)}$ is a relatively compact subset of $L^{2}(\Omega, H)$. The differentiability properties of $\mathbf{p} \in L^{2}\left(\Omega, \mathbf{R}^{3}\right) \mapsto \mathbf{k}[\mathbf{p}] \in L^{2}\left(\Omega, \mathbf{R}^{3}\right)$ stated in Hypotheses (H2)ii-iii then yield

$$
\mathbf{k}[\tilde{\mathbf{m}}(\xi+\cdot)]-\mathbf{k}[\mathbf{m}]=D \mathbf{k}[\mathbf{m}] \cdot(\xi \cdot \nabla \mathbf{m})+o(|\xi|)
$$

in $L^{2}(\Omega, H)$. This leads to

$$
\frac{\mathbf{k}\left[\mathbf{m}_{s t}^{\theta}\right]-\mathbf{k}\left[\mathbf{m}_{s t}^{-\theta}\right]}{t}-2 s D \mathbf{k}[\mathbf{m}] \cdot(\theta \cdot \nabla \mathbf{m}) \stackrel{t \downarrow 0}{\longrightarrow} 0 \quad \text { in } L^{2}(\Omega, H) \text { uniformly in }(\theta, s) \in \mathbb{S}^{d-1} \times(0,1) .
$$

Integrating in $(\theta, s) \in \mathbb{S}^{d-1} \times(0,1)$, we obtain

$$
R_{1}(t) \stackrel{t \downarrow 0}{\longrightarrow} \frac{1}{2} \int_{\mathbb{S}^{d-1}} \int_{\Omega}\{D \mathbf{k}[\mathbf{m}] \cdot(\theta \cdot \nabla \mathbf{m})\}(x) \cdot(\theta \cdot \nabla \mathbf{m})(x) d x d \mathcal{H}^{d-1}(\theta) .
$$

And since $\int_{\mathbb{S}^{d-1}}(\theta \otimes \theta) d \mathcal{H}^{2}(\theta)=\left(\left|\mathbb{S}^{d-1}\right| / d\right) \mathrm{Id}$, we get

$$
R_{1}(t) \stackrel{t \downarrow 0}{\longrightarrow} \frac{\left|\mathbb{S}^{d-1}\right|}{2 d} \int_{\Omega} \sum_{i=1}^{d}\left\{D \mathbf{k}[\mathbf{m}] \cdot\left(\partial_{i} \mathbf{m}\right)\right\} \cdot \partial_{i} \mathbf{m} .
$$

Next, in order to evaluate $R_{2}(t)$, we first integrate by parts to get

$$
\begin{aligned}
R_{2}(t)= & -\int_{\mathbb{S}^{d-1}} \int_{0}^{1} \int_{\Omega}\left(\theta \cdot \nabla\left\{\mathbf{k}\left[\mathbf{m}_{s t}^{\theta}\right]\right\}\right)(x) \cdot \frac{\left\{\mathbf{m}_{s t}^{\theta}-\mathbf{m}\right\}(x)}{t} d x d s d \mathcal{H}^{d-1}(\theta) \\
& +\int_{\mathbb{S}^{d-1}} \int_{0}^{1} \int_{\partial \Omega} \mathbf{k}\left[\mathbf{m}_{s t}^{\theta}\right](y) \cdot \frac{\left\{\mathbf{m}_{s t}^{\theta}-\mathbf{m}\right\}(y)}{t}(\theta \cdot \mathbf{n}(y)) d \mathcal{H}^{2}(y) d s d \mathcal{H}^{d-1}(\theta) \\
=: & R_{2,1}(t)+R_{2,2}(t) .
\end{aligned}
$$

The expansion $\mathbf{m}_{s t}^{\theta}-\mathbf{m}=s t \theta \cdot \nabla \mathbf{m}+o(t)$ in $L^{2}(\Omega, H)$ being valid uniformly in $(\theta, s) \in \mathbb{S}^{d-1} \times(0,1)$ we obtain by Hypothesis (H2)-iv.:

$$
R_{2,1}(t) \stackrel{t \downarrow 0}{\longrightarrow}-\frac{1}{2} \int_{\mathbb{S}^{d-1}} \int_{\Omega}(\theta \cdot \nabla\{\mathbf{k}[\mathbf{m}]\}) \cdot(\theta \cdot \nabla \mathbf{m}) d \mathcal{H}^{d-1}(\theta)=-\frac{\left|\mathbb{S}^{d-1}\right|}{2 d} \int_{\Omega} \sum_{i=1}^{d} \partial_{i}\{\mathbf{k}[\mathbf{m}]\} \partial_{i} \mathbf{m} .
$$

We now establish that the boundary term $R_{2,2}(t)$ goes to 0 as $t \downarrow 0$. Then (39) will follow from (41), (42), (43) and the local optimality of $\mathbf{m}$.

Writing $\mathbf{m}_{s t}^{\theta}-\mathbf{m}=s t \int_{0}^{1} \theta \cdot \nabla \mathbf{m}_{r s t} d r$, we get

$$
R_{2,2}(t)=\int_{\mathbb{S}^{d-1}} \int_{0}^{1} s \int_{\partial \Omega} \int_{0}^{1} \mathbf{k}\left[\mathbf{m}_{s t}^{\theta}\right] \cdot\left(\theta \cdot \nabla \mathbf{m}_{r s t}^{\theta}\right)(\theta \cdot \mathbf{n}) d r d \mathcal{H}^{d-1} d s d \mathcal{H}^{d-1}(\theta) .
$$

Using Fubini and the change of variables $r=q / s$, we compute,

$$
R_{2,2}(t)=\int_{\mathbb{S}^{d-1}} \int_{\partial \Omega}\left[\int_{(0,1)^{2}} \mathbf{k}\left[\mathbf{m}_{s t}^{\theta}\right] \cdot\left(\theta \cdot \nabla \mathbf{m}_{q t}^{\theta}\right)(\theta \cdot \mathbf{n}) d q d s\right] d \mathcal{H}^{d-1} d \mathcal{H}^{d-1}(\theta) .
$$


By Hypothesis (H2)iv and the trace Theorem, the mapping $\xi \in B_{t^{\star}}(0) \mapsto \mathbf{k}[\mathbf{p}]_{\mid \partial \Omega} \in L^{2}(\partial \Omega, H)$ is continuous. In particular,

$$
\left\{(y, s) \mapsto \mathbf{k}\left[\mathbf{m}_{s t}^{\theta}\right](y)\right\} \stackrel{t \downarrow 0}{\longrightarrow} \mathbf{k}[\mathbf{m}] \quad \text { in } L^{2}(\partial \Omega \times(0,1)) \quad \text { uniformly in } \theta \in \mathbb{S}^{d-1} .
$$

On the other hand, by Lemma 28, we also have

$$
\left\{(y, q) \mapsto\left(\theta \cdot \nabla \mathbf{m}_{q t}^{\theta}\right)(y)\right\} \stackrel{t \downarrow 0}{\longrightarrow} \mathbf{k}[\mathbf{m}] \quad \text { in } L^{2}(\partial \Omega \times(0,1)) \quad \text { uniformly in } \theta \in \mathbb{S}^{d-1} .
$$

Consequently, $R_{2,2}(t)$ converges, as $t \downarrow 0$, towards

$$
\int_{\partial \Omega} \mathbf{k}[\mathbf{m}] \cdot\left(\int_{\mathbb{S}^{d-1}}\left(\theta \cdot \nabla \mathbf{u}_{0}\right)(\theta \cdot \mathbf{n}) d \mathcal{H}^{2}(\theta)\right) d \mathcal{H}^{2}=\frac{\left|\mathbb{S}^{d-1}\right|}{d} \int_{\partial \Omega} \mathbf{k}[\mathbf{m}] \cdot\left(\mathbf{n} \cdot \nabla \mathbf{u}_{0}\right) d \mathcal{H}^{2}=0 .
$$

For the last identity, we recall that $\mathbf{u}_{0}$ is defined as an element of $H^{1}\left(\partial \Omega, \mathbf{R}^{3}\right)$, so $\mathbf{n} \cdot \nabla \mathbf{u}_{0} \equiv 0$ on $\partial \Omega$. This ends the proof of the Lemma.

We are now able to establish the first part of Theorem 14. By Hypothesis (H1), $\Omega$ is uniformly convex and $0 \in \Omega$, so there exists $c_{\Omega}>0$ such that

$$
c_{\Omega} a_{y}(\xi) \geq(y \cdot \mathbf{n}(y))|\xi|^{2}, \quad \text { for every } y \in \partial \Omega, \xi \in T_{y} \partial \Omega .
$$

Lemmas 30 and 31 then lead to

$$
\mathcal{D}(\mathbf{m}) \leq \varepsilon^{2}\left\{L(\mathbf{m})+L^{\prime}(\mathbf{m})+Q(\mathbf{m})\right\} .
$$

with

$$
\begin{gathered}
L(\mathbf{m}):=\int_{\Omega} \mathbf{k}[\mathbf{m}](x) \cdot(x \cdot \nabla \mathbf{m})(x) d x \\
\left.Q(\mathbf{m}):=c_{\Omega} \int_{\Omega} \sum_{i=1}^{3}\left(D \mathbf{k}[\mathbf{m}] \cdot \partial_{i} \mathbf{m}\right\} \cdot \partial_{i} \mathbf{m}\right), \quad L^{\prime}(\mathbf{m}):=-c_{\Omega} \int_{\Omega} \sum\left(\partial_{i}\{\mathbf{k}[\mathbf{m}]\} \cdot \partial_{i} \mathbf{m}\right) .
\end{gathered}
$$

Using the bounds of Hypothesis (H2) we have:

$$
L(\mathbf{m}) \leq C_{1}\|\nabla \mathbf{m}\|_{L^{2}}, \quad L^{\prime}(\mathbf{m}) \leq c_{\Omega} C_{3}\|\nabla \mathbf{m}\|_{L^{2}}\left(1+\|\nabla \mathbf{m}\|_{L^{2}}, \quad Q(\mathbf{m}) \leq c_{\Omega} C_{2}\|\nabla \mathbf{m}\|_{L^{2}}^{2} .\right.
$$

Plugging these estimates in (45), and simplifying, we obtain

$$
\|\nabla \mathbf{m}\|_{L^{2}} \leq C_{\mathcal{F}} \varepsilon^{2} \quad \text { for } \varepsilon<\varepsilon_{\mathcal{F}},
$$

with

$$
\varepsilon_{\mathcal{F}}:=\frac{1}{2 \sqrt{c_{\Omega}\left(C_{2}+C_{3}\right)}}, \quad C_{\mathcal{F}}:=4\left(C_{1}+c_{\Omega} C_{3}\right) .
$$

This establishes Theorem 14.i.

\subsection{Proof of Theorem 14. ii. (target variations)}

Let us now assume that hypotheses (H3-H4) also hold. We show that in this case, the right hand side of (45) is bounded by $C \varepsilon^{2}\|\nabla \mathbf{m}\|_{L^{2}}^{2}$. We already have the desired quadratic estimate for $Q(t)$ (last inequality of (46)). Next, by Hypothesis (H3) we also have

$$
\left|L^{\prime}(\mathbf{m})\right| \leq c_{\Omega} C_{3}^{\prime}\|\nabla \mathbf{m}\|_{L^{2}}^{2} .
$$

The most difficult part is to establish that there exist $\varepsilon_{L}>0$ and $C_{L} \geq 0$ depending on $\Omega$ and $\mathcal{F}$ such that for $0<\varepsilon<\varepsilon_{l}$, we have:

$$
|L(\mathbf{m})| \leq C_{L}\|\nabla \mathbf{m}\|_{L^{2}}^{2}
$$

Taking this estimate for granted, we end the proof as follows. Using (47), (48) and the last estimate of (46) to bound the right hand side of (45), we get, for $\varepsilon<\varepsilon_{L}$,

$$
\left(1-2\left(c_{\Omega}\left(C_{2}+C_{3}^{\prime}\right)+C_{L}\right) \varepsilon^{2}\right) \mathcal{D}(\mathbf{m}) \leq 0 .
$$


Thus $\mathbf{m}$ is constant as soon as $\varepsilon<\varepsilon_{\mathcal{F}}^{\prime}:=\min \left(\varepsilon_{L}, 1 / \sqrt{2\left(c_{\Omega}\left(C_{2}+C_{3}^{\prime}\right)+C_{L}\right)}\right)$.

To end the proof we establish (48). Let us first write $L(\mathbf{m})=L_{1}(\mathbf{m})+L_{2}(\mathbf{m})$ with

$$
L_{1}(\mathbf{m}):=\mathbf{k}[\langle\mathbf{m}\rangle] \cdot \int_{\Omega}(x \cdot \nabla \mathbf{m}) d x, \quad L_{2}(\mathbf{m}):=\int_{\Omega}\{\mathbf{k}[\mathbf{m}]-\mathbf{k}[\langle\mathbf{m}\rangle]\} \cdot(x \cdot \nabla \mathbf{m}) d x,
$$

where, with an abuse of notation, we identify $\mathbf{k}[\mathbf{m}]$ with its constant value $\mathbf{N}_{\text {eff }} \cdot\langle\mathbf{m}\rangle+\nabla \psi(\langle\mathbf{m}\rangle) \in H$ inside $\Omega$. For the second term, we use the Cauchy-Schwarz inequality and the differentiability of $\mathbf{p} \in L^{2}(\Omega, H) \mapsto \mathbf{k}[\mathbf{p}] \in$ $L^{2}(\Omega, H)$ with the estimate of Hypothesis (H2)-ii to get

$$
\left|L_{2}(\mathbf{m})\right| \leq C_{2}\|\mathbf{m}-\langle\mathbf{m}\rangle\|_{L^{2}}\|\nabla \mathbf{m}\|_{L^{2}} \leq C_{2} C_{P}\|\nabla \mathbf{m}\|_{L^{2}}^{2} .
$$

Let us now bound $L_{1}(\mathbf{m})$. Let $\sigma \in H$ be a projection of $\langle\mathbf{m}\rangle$ on $\mathcal{S}$, i.e. $\sigma \in \operatorname{argmin}\left\{\left|\sigma^{\prime}-\langle\mathbf{m}\rangle\right|^{2} ; \sigma^{\prime} \in \mathcal{S}\right\}$. First, by definition of $\sigma$ (with $\sigma^{\prime}=\mathbf{m}(x) \in \mathcal{S}$ ) we have:

$$
|\sigma-\langle\mathbf{m}\rangle|^{2}=\frac{1}{|\Omega|} \int_{\Omega}|\sigma-\langle\mathbf{m}\rangle|^{2} \leq \frac{1}{|\Omega|} \int_{\Omega}|\mathbf{m}(x)-\langle\mathbf{m}\rangle|^{2} d x \leq \frac{C_{P}^{2}}{|\Omega|}\|\nabla \mathbf{m}\|_{L^{2}}^{2} .
$$

By the triangular inequality this leads to

$$
\|\sigma-\mathbf{m}\|_{L^{2}} \leq 2 C_{P}\|\nabla \mathbf{m}\|_{L^{2}} .
$$

Now, we integrate by parts to obtain

$$
L_{1}(\mathbf{m})=d|\Omega| \mathbf{k}[\langle\mathbf{m}\rangle] \cdot\left(\langle\mathbf{m}\rangle_{\partial}-\langle\mathbf{m}\rangle\right), \quad \text { with } \quad\langle\mathbf{m}\rangle_{\partial}:=\frac{1}{d|\Omega|} \int_{\partial \Omega} \mathbf{m}(y)(y \cdot \mathbf{n}(y)) d \mathcal{H}^{d-1}(y) .
$$

Let us perform the orthogonal decomposition:

$$
\langle\mathbf{m}\rangle_{\partial}-\langle\mathbf{m}\rangle=: \xi_{\mathbf{m}}+\zeta_{\mathbf{m}}, \quad \text { with } \quad \xi_{\mathbf{m}} \in N_{\sigma} \mathcal{S}, \zeta_{\mathbf{m}} \in T_{\sigma} \mathcal{S} .
$$

Let $\xi:=\xi_{\mathbf{m}} /\left|\xi_{\mathbf{m}}\right| \in N_{\sigma} \mathcal{S}$. Using hypothesis (H4) and (51) we have

$$
|(\langle\mathbf{m}\rangle-\sigma) \cdot \xi|=\left|\frac{1}{|\Omega|} \int_{\Omega}[\mathbf{m}(x)-\sigma] \cdot \xi d x\right| \leq \frac{C_{\mathcal{S}}^{\prime}}{|\Omega|} \int_{\Omega}|\mathbf{m}(x)-\sigma|^{2} d x \leq \frac{4 C_{\mathcal{S}}^{\prime} C_{P}^{2}}{|\Omega|}\|\nabla \mathbf{m}\|_{L^{2}}^{2} .
$$

We have a similar estimate for $\left|\langle\mathbf{m}\rangle_{\partial}-\sigma\right|$ which leads to,

We conclude that

$$
\left|\xi_{\mathbf{m}}\right| \leq \frac{2 C_{\mathcal{S}}^{\prime}\left(6 C_{P}^{2}+C_{P}^{\prime 2}\right)}{|\Omega|}\|\nabla \mathbf{m}\|_{L^{2}}^{2}
$$

$$
|d| \Omega\left|\mathbf{k}[\langle\mathbf{m}\rangle] \cdot \xi_{\mathbf{m}}\right| \leq 2 d C_{1} C_{\mathcal{S}}^{\prime}\left(6 C_{P}^{2}+C_{P}^{\prime 2}\right)\|\nabla \mathbf{m}\|_{L^{2}}^{2}
$$

We now bound the term $d|\Omega| \mathbf{k}[\langle\mathbf{m}\rangle] \cdot \zeta_{\mathbf{m}}$. First, we have an obvious linear control of $\zeta_{\mathbf{m}}$ :

$$
\left|\zeta_{\mathbf{m}}\right| \leq\left|\langle\mathbf{m}\rangle_{\partial}-\langle\mathbf{m}\rangle\right| \leq \frac{C_{P}^{\prime}}{\sqrt{d|\Omega|}}\|\nabla \mathbf{m}\|_{L^{2}}
$$

Eventually, we use the optimality of $\mathbf{m}$ to establish that the following estimate holds

$$
|\Omega||\mathbf{k}[\langle\mathbf{m}\rangle] \cdot \zeta| \leq\left(2 C_{1} C_{\mathcal{S}}+C_{2}\right) C_{P}\|\nabla \mathbf{m}\|_{L^{2}}|\zeta| \quad \text { for every } \zeta \in T_{\sigma} \mathcal{S} .
$$

Let $\zeta \in T_{\sigma} \mathcal{S}$. By hypothesis (H4) there exists a smooth one parameter group of isometries of $\mathcal{S},\{R(t)\}_{t \in \mathbf{R}}$, such that $\dot{R}(0) \cdot \sigma=\zeta$ and $\|\dot{R}(0)\|_{\infty} \leq C_{\mathcal{S}}|\zeta|$.

Let us set $\gamma_{t}:=R(t) \cdot \mathbf{m}$, for $t \in \mathbf{R}$. By local optimality of $\mathbf{m}$ the function $f(t):=\mathcal{F}\left(\gamma_{t}\right)$ admits a local minimum at $t=0$. In particular,

$$
0=f^{\prime}(0)=D \mathcal{F}(\mathbf{m}) \cdot\{\dot{R}(0) \cdot \mathbf{m}\}=\int_{\Omega} \mathbf{k}[\mathbf{m}](x) \cdot(\dot{R}(0) \cdot \mathbf{m}(x)) d x
$$


Now, let us write

$$
\begin{aligned}
|\Omega| \mathbf{k}[\langle\mathbf{m}\rangle] \cdot \zeta & =\int_{\Omega} \mathbf{k}[\langle\mathbf{m}\rangle] \cdot \zeta \\
& =\int_{\Omega}\{\mathbf{k}[\langle\mathbf{m}\rangle]-\mathbf{k}[\mathbf{m}]\} \cdot \zeta+\int_{\Omega} \mathbf{k}[\mathbf{m}] \cdot\{\dot{R}(0) \cdot(\sigma-\mathbf{m})\}+\int_{\Omega} \mathbf{k}[\mathbf{m}] \cdot \dot{R}(0) \cdot \mathbf{m} .
\end{aligned}
$$

By (55), the last term vanishes. Using the Cauchy Schwarz inequality and (51), the second term satisfies the estimate

$$
\left|\int_{\Omega} \mathbf{k}[\mathbf{m}] \cdot\{\dot{R}(0) \cdot(\sigma-\mathbf{m})\}\right| \leq 2 C_{1} C_{\mathcal{S}} C_{P}\|\nabla \mathbf{m}\|_{L^{2}}|\zeta|,
$$

while for the first term, we have

$$
\left|\int_{\Omega}\{\mathbf{k}[\langle\mathbf{m}\rangle]-\mathbf{k}[\mathbf{m}]\} \cdot \zeta\right| \leq C_{2}\|\langle\mathbf{m}\rangle-\mathbf{m}\|_{L^{2}}|\zeta| \leq C_{2} C_{P}\|\nabla \mathbf{m}\|_{L^{2}}|\zeta| .
$$

The last two inequalities imply (54) which together with (52) and (53) yield $\left|L_{1}(\mathbf{m})\right| \leq C\|\nabla \mathbf{m}\|_{L^{2}}^{2}$ for some $C \geq 0$ depending on $d,|\Omega|, C_{1}, C_{2}, C_{\mathcal{S}}^{\prime}$, and $C_{\mathcal{S}}$. This ends the proof of Theorem 14 .

\section{Concluding remarks and further generalizations.}

Let us discuss how our results depend on the shape of the domain $\Omega$. Paying attention to the constants in the estimates, we see that the parameters $C_{\mathcal{F}}, \varepsilon_{\mathcal{F}}$ and $\varepsilon_{\mathcal{F}}^{\prime}$ in Theorem 14. only depend on $c_{\Omega}$, the Poincare constant $C_{P}, C_{P}^{\prime}$ and the constants $C_{1}, C_{2}$ and $C_{3}$ of Hypothesis (H2). The Poincaré constant is uniformly bounded since $\Omega$ is a convex domain with unit diameter.

In the context of micromagnetism (Theorem 8 ) the constants $C_{1}$ and $C_{2}$ only depend on $\psi$, but $C_{3}$ also depends on the constant $C_{3}^{\prime}$ of Proposition 17 for which we do not have an explicit bound. It would be interesting to know whether this constant admits a uniform bound in the set of smooth convex domains.

The constant $c_{\Omega}$ (introduced in (44)) is the inverse of the minimal curvature of $\partial \Omega$. In particular, this constant blows up when considering a sequence of unit diameter convex domains $\left(\Omega_{k}\right)_{k}$ such that $\Omega_{k}$ is included in the thin cylinder $B_{\mathbf{R}^{2}}(0,1) \times(-1 / k, 1 / k)$. This includes the case of thin ellipsoids. So, our result degenerates in the limit of thin ellipsoids.

We do not claim that the uniform convexity assumption on the domain is sharp. However, we believe that the results do not hold in some complex geometries. For example, if $\Omega \subset \mathbf{R}^{3}$ is the ball with cavity,

$$
\Omega=\left\{x \in \mathbf{R}^{3} ; 1<|x|<2\right\}
$$

then we believe that the non-constant mapping $\mathbf{m}(x):=x /|x|$ is a local minimizer of $\mathcal{D}$ in $H^{1}\left(\Omega, \mathbb{S}^{2}\right)$. In the perturbed case, we may conjecture that for $\varepsilon$ small enough, we can find in the neighborhood of the set $\left\{R \mathbf{m}: R \in \mathrm{SO}_{3}(\mathbf{R})\right\}$ some local minimizers of $\mathcal{D}_{\varepsilon}$ in $H^{1}\left(\Omega, \mathbb{S}^{2}\right)$.

In the proof of Theorem 9, we only test the local optimality of $\mathbf{m}$ under a small set of variations: small dilations of the domain (for which we only need a first order optimality condition) and small translations of the domain. The nature of the target set $\mathcal{S}$ does not play any role in the proof.

In the proof of Theorem 14.ii, we also use the optimality of $\mathbf{m}$ with respect to the target set. If we denote by $\left\{\mathbf{m}_{t}\right\}_{t \in\left(-t^{\prime}, t^{\prime}\right)} \subset H^{1}(\Omega, \mathcal{S})$ the trajectory corresponding to one of these variations, we require that $\mathcal{D}_{\varepsilon}(\mathbf{m}) \leq \mathcal{D}_{\varepsilon}\left(\mathbf{m}_{t}\right)$ for $|t|<t^{\prime}$ small enough. In the case of domain translations, we also used that this property holds uniformly in every direction of translation. We do this when integrating the optimality condition on the set of directions $\theta \in \mathbb{S}^{d-1}$ in (26), (40). We could avoid this if we already knew that $\mathbf{m}$ were smooth, in this case we would prove the counterpart of (27) without integrating in $\theta$. In this situation, we could weaken the optimality hypothesis to

$$
\mathcal{D}_{\varepsilon}(\mathbf{m}) \leq \lim _{t \downarrow 0} \mathcal{D}_{\varepsilon}\left(\mathbf{m}_{t}\right), \quad \forall\left\{\mathbf{m}_{t}\right\} \in C^{1}\left(\left[0, t^{\prime}\right), L^{2}\left(\Omega, \mathbb{S}^{2}\right)\right), \mathbf{m}_{0}=\mathbf{m} \text { and } \frac{d}{d t} \mathbf{m}_{\mid t=0} \neq 0 .
$$


We did not succeed in providing a proof with this weaker assumption in the general case. Let us mention however that when $\Omega$ is the unit open ball centered at 0 , then $\mathbf{n}(y)=y$ on $\partial \Omega$ and we can use the expansions $\overline{\mathbf{m}}$ of $\mathbf{m}$ in place of the expansion $\tilde{\mathbf{m}}$ (see their definitions in (19), (25)). In this case, the proof simplifies: we only need to consider domain translations in the directions $\mathbf{e}_{1}, \ldots, \mathbf{e}_{d}$, so that Theorem 14 holds under the weaker assumption (56).

Theorems 9 and 14 may be generalized. First, if $M$ is an invertible matrix of $\mathbf{R}^{d \times d}$, we see that, by the change of variable $z=M x$, Theorem 9 holds for the functional

$$
\mathcal{E}_{M}(\mathbf{m}):=\frac{1}{p} \int|M \nabla \mathbf{m}|^{p}
$$

We may also consider small perturbations of the form,

$$
\mathcal{E}_{\eta}(\mathbf{m}):=\frac{1}{p} \int_{\Omega}|\nabla \mathbf{m}|^{p}+\eta \int_{\Omega} a(x, \nabla \mathbf{m})
$$

with $\eta \in \mathbf{R}$ and $a \in C^{2,1}\left(\bar{\Omega} \times H^{d}, \mathbf{R}\right)$ such that

$$
\sup _{(x, \mathbf{v}) \Omega \times H} \frac{\left(\left|D_{x} a\right|+\left|D_{x}^{2} a\right|+|\mathbf{v}|\left|D_{\mathbf{v}} a\right|\right)(x, \mathbf{v})}{|\mathbf{v}|^{p}}<\infty .
$$

We obtain, with reasonable modifications of the current proof that Theorem 9 holds for $\mathcal{E}_{\eta}$ under the condition $|\eta|<\eta_{c}$ where $\eta_{c}>0$ depends on $c_{\Omega}$ and $a$.

\section{Appendix A (proof of Proposition 13)}

Let $\Omega \subset \mathbf{R}^{d}$ be a bounded convex smooth open set with diameter $\delta>0$ and assume that $0 \in \partial \Omega$. We consider a real valued function $f \in C^{\infty}(\bar{\Omega})$, (the result for $f \in H^{1}(\Omega)$ is obtained by density of $C^{\infty}(\bar{\Omega})$ in $H^{1}(\Omega)$ and by continuity of the trace mapping $f \in H^{1}(\Omega) \mapsto f_{\mid \partial \Omega} \in L^{2}(\partial \Omega)$ ). We have to estimate the quantity

$$
I(f):=\int_{\Omega} \int_{\partial \Omega}|f(x)-f(y)|^{2}(\mathbf{n}(y) \cdot y) d \mathcal{H}^{d-1}(y) d x .
$$

For $y \in \partial \Omega$, we define te following weighted mean value of $f$ along the segment $(0,1) y$ :

$$
\langle f\rangle_{y}:=\frac{d+1}{2} \int_{0}^{1} r^{\frac{d-1}{2}} f(r y) d r .
$$

We then decompose $f(y)$ as $\langle f\rangle_{y}+\left[f(y)-\langle f\rangle_{y}\right]$ to get $I(f) \leq 2\left(|\Omega| I_{1}(f)+I_{2}(f)\right)$, with

$$
\begin{aligned}
I_{1}(f):=\int_{\partial \Omega}\left|f(y)-\langle f\rangle_{y}\right|^{2}(\mathbf{n}(y) \cdot y) d \mathcal{H}^{d-1}(y), \\
I_{2}(f):=\int_{\Omega} \int_{\partial \Omega}\left|f(x)-\langle f\rangle_{y}\right|^{2}(\mathbf{n}(y) \cdot y) d \mathcal{H}^{d-1}(y) d x .
\end{aligned}
$$

We start by estimating $I_{1}(f)$. Let us fix $y \in \partial \Omega$, we have,

$$
\begin{aligned}
f(y)-\langle f\rangle_{y} & =\frac{(d+1)}{2} \int_{0}^{1} r^{\frac{d-1}{2}}(f(y)-f(r y)) d r \\
& =\frac{(d+1)}{2} \int_{0}^{1} r^{\frac{d-1}{2}}(1-r) \int_{0}^{1} y \cdot \nabla f((r+(1-r) s) y) d s d r
\end{aligned}
$$

Using the change of variable $s=(t-r) /(1-r)$ and then Fubini, we obtain,

$$
f(y)-\langle f\rangle_{y}=\frac{(d+1)}{2} \int_{0}^{1} r^{\frac{d-1}{2}} \int_{r}^{1} y \cdot \nabla f(t y) d t d r=\int_{0}^{1} y \cdot \nabla f(t y)^{\frac{d+1}{2}} d t .
$$


Squaring and using the Jensen inequality, we get:

$$
\left|f(y)-\langle f\rangle_{y}\right|^{2} \leq|y|^{2} \int_{0}^{1}|\nabla f(t y)|^{2} t^{d+1} d t \leq \delta^{2} \int_{0}^{1}|\nabla f(t y)|^{2} t^{d-1} d t .
$$

Then, we multiply by $(y \cdot \mathbf{n}(y))$ and integrate in $y \in \partial \Omega$. Using the change of variable $z=\psi(y, t):=t y$, which maps $\partial \Omega \times(0,1)$ onto $\Omega \backslash\{0\}$, we get

$$
I_{1}(f) \leq \delta^{2} \int_{\Omega}|\nabla f|^{2}(z) \frac{[t(z)]^{d-1}(y(z) \cdot \mathbf{n}(y(z)))}{J_{\psi}\left(\psi^{-1}(z)\right)} d z=\delta^{2} \int_{\Omega}|\nabla f|^{2}(z) d z
$$

with the notation, $\psi^{-1}(z)=:(y(z), t(z))$ and $J_{\psi}(y, t)=\sqrt{\operatorname{det} D \psi^{T} \cdot D \psi}(y, t)$. Indeed, introducing the orthogonal decomposition $\mathbf{R}^{d}=T_{y} \partial \Omega \oplus \mathbf{R} \mathbf{n}(y) \simeq T_{y} \partial \Omega \oplus \mathbf{R}$, we compute the Jacobian matrix of $\psi$ in these spaces:

$$
D \psi(y, t)=\left(\begin{array}{cc}
t \operatorname{Id}_{T_{y} \partial \Omega} & (y-(y \cdot \mathbf{n}(y)) \mathbf{n}(y)) \\
0 & (y \cdot \mathbf{n}(y))
\end{array}\right) .
$$

The Jacobian determinant of $\psi$ is $J_{\psi}(y, s)=t^{d-1}(y \cdot \mathbf{n}(y))$.

Now we bound $I_{2}(f)$. We first use the definition of $\langle f\rangle_{y}$ and the Cauchy-Schwarz inequality to get for every $(x, y) \in \Omega \times \partial \Omega$ :

$$
\left|f(x)-\langle f\rangle_{y}\right|^{2} \leq \frac{(d+1)^{2}}{4} \int_{0}^{1}|f(x)-f(r y)|^{2} r^{d-1} d r
$$

Integrating in $y \in \partial \Omega$, and using the change of variable $z=\psi(y, r)$ as above, we obtain (after integration in $x \in \Omega)$ :

$$
I_{2}(f) \leq \frac{(d+1)^{2}}{4} \int_{\Omega \times \Omega}|f(x)-f(z)|^{2} d x d z \leq(d+1)^{2}|\Omega| C_{P}^{2}\|\nabla f\|_{L^{2}}^{2} .
$$

Inequality (3) follows from (57) and (58) with

$$
\frac{C_{P}^{\prime}}{\delta}=\sqrt{2\left[1+(d+1)^{2}\left(\frac{C_{P}}{\delta}\right)^{2}\right]} \leq \sqrt{2}\left(1+\frac{(d+1) C_{P}}{\delta}\right) .
$$

Since $C_{P} / \delta \leq 1 / \pi$, we have $C_{P}^{\prime} / \delta \leq \sqrt{2}(1+(d+1) / \pi)$ as claimed.

\section{Acknowledgements}

The first and last authors acknowledge support from the ANR project ANR-08- BLAN-0199-01 of the French Ministry of Research, "Micromagnetism: Mathematics Applied to New Physical Interactions".

\section{Bibliography}

[1] A. Aharoni. Elongated single-domain ferromagnetic particles. J. Appl. Phys., 63(12):0, 1988.

[2] F. Alouges and K. Beauchard. Magnetization switching on small ferromagnetic ellipsoidal samples. ESAIM Control Optim. Calc. Var., 15(3):0, 2009.

[3] F. Alouges, S. Conti, A. DeSimone and Y. Pokern. Energetics and switching of quasi-uniform states in small ferromagnetic particles. M2AN, 38(2):235-248, 2004.

[4] F. Alouges, S. Faure and J. Steiner. The vortex core structure inside spherical ferromagnetic particles. Disc. Cont. Dyn. Syst. A, 27(4):1259-1283, 2010.

[5] M. Bebendorf. A note on the Poincaré inequality for convex domains. Z. Anal. Anwendungen, 22(4):0, 2003.

[6] W. F. Brown. The fundamental theorem of the theory of fine ferromagnetic particles. J. Appl. Phys., 39:0, 1968.

[7] W. F. Brown. Magnetostatic Principles in Ferromagnetism. North-Holland Publishing Co., 1962. 
[8] G. Carbou and P. Fabrie. Regular solutions for Landau-Lifschitz equation in a bounded domain. Differential Integral Equations, 14(2):0, 2001.

[9] R. P. Cowburn and M. E. Welland. Micromagnetics of the single-domain state of square ferromagnetic nanostructures. Phys. Rev. B, 58:0, 1998.

[10] A. DeSimone. Hysteresis and imperfection sensitivity in small ferromagnetic particles. Meccanica, 30(5):0, 1995. Microstructure and phase transitions in solids (Udine, 1994).

[11] G. Di Fratta, C. Serpico and M. D'Aquino. A generalization of the fundamental theorem of brown for fine ferromagnetic particles. Physica B, 407(9):0, 2012.

[12] J. Eells and L. Lemaire. A report on harmonic maps. Bull. London Math. Soc., 10(1):0, 1978.

[13] J. Eells and L. Lemaire. Another report on harmonic maps. Bull. London Math. Soc., 20(5):0, 1988.

[14] J. Eells and L. Lemaire. Selected topics in harmonic maps, volume 50 of CBMS Regional Conference Series in Mathematics. Published for the Conference Board of the Mathematical Sciences, Washington, DC, 1983.

[15] James Eells and Luc Lemaire. Two reports on harmonic maps. World Scientific Publishing Co. Inc., River Edge, NJ, 1995.

[16] M. Fuchs. P-harmonic obstacle problems. I. Partial regularity theory. Ann. Mat. Pura Appl. (4), 156:0, 1990.

[17] M. Fuchs. The blow-up of p-harmonic maps. Manuscripta Math., 81(1-2):0, 1993.

[18] R. Hardt, D. Kinderlehrer and F.-H. Lin. Stable defects of minimizers of constrained variational principles. Ann. Inst. H. Poincaré Anal. Non Linéaire, 5(4):0, 1988.

[19] R. M. Hardt. Singularities of harmonic maps. Bull. Amer. Math. Soc. (N.S.), 34(1):0, 1997.

[20] A. Hubert and R. Schäfer. Magnetic Domains. Springer-Verlag, 1998.

[21] F. Hélein and J. C. Wood. Harmonic maps. In Handbook of global analysis, page 0. Elsevier Sci. B. V., Amsterdam, 2008.

[22] O. D. Kellogg. Foundations of Potential Theory. Grundlehren der mathematischen Wissenschaften. Springer Verlag, 1967.

[23] S. Luckhaus. Partial Hölder continuity for minima of certain energies among maps into a Riemannian manifold. Indiana Univ. Math. J., 37(2):0, 1988.

[24] C. B. Morrey Jr. The problem of Plateau on a Riemannian manifold. Ann. of Math. (2), 49:0, 1948.

[25] J. C. Maxwell. A treatise on electricity and magnetism. Vol. 2. Oxford Classic Texts in the Physical Sciences. The Clarendon Press Oxford University Press, New York, 1998. Reprint of the third (1891) edition.

[26] J. A. Osborn. Demagnetizing factors of the general ellipsoid. Phys. Rev., 67(11,12):0, 1945.

[27] L. E. Payne and H. F. Weinberger. An optimal Poincaré inequality for convex domains. Arch. Rational Mech. Anal., 5:0, 1960.

[28] R. Schoen and K. Uhlenbeck. A regularity theory for harmonic maps. J. Differential Geom., 17(2):0, 1982.

[29] V. V. Slastikov. A note on configurational anisotropy. Proc. R. Soc. Lond. Ser. A Math. Phys. Eng. Sci., 466(2123):0, 2010.

[30] E. C. Stoner and E. P. Wohlfarth. A mechanism of magnetic hysteresis in heterogeneous alloys. Phil. Trans. R. Soc. London Ser. A, 240:0, 1948.

[31] L. Simon. Lectures on geometric measure theory, volume 3 of Proceedings of the Centre for Mathematical Analysis, Australian National University. Australian National University Centre for Mathematical Analysis, Canberra, 1983. 